\title{
Transcriptional Regulator ZEB2 Is Essential for Bergmann Glia Development
}

\author{
๑Li He, ${ }^{1,2 *}$ Kun Yu, ${ }^{1 *}$ Fanghui Lu, ${ }^{6}$ Jiajia Wang, ${ }^{3}$ Laiman N. Wu, ${ }^{2}$ Chuntao Zhao, ${ }^{2}$ Qianmei Li, ${ }^{1}{ }^{\odot X i a n y a o ~ Z h o u, ~}{ }^{1}$ \\ Hanmin Liu, ${ }^{1}$ Dezhi Mu, ${ }^{1}$ Mei Xin, ${ }^{2}$ Mengsheng Qiu, ${ }^{4,5}$ and ${ }^{\mathbb{Q}}$. Richard $\mathrm{Lu}^{1,2}$ \\ ${ }^{1}$ Key Laboratory of Birth Defects and Related Diseases of Women and Children of Ministry of Education, Department of Pediatrics, West China Second \\ University Hospital, Sichuan University, Chengdu 610041, China, ${ }^{2}$ Department of Pediatrics, Division of Experimental Hematology and Cancer Biology, \\ Cincinnati Children's Hospital Medical Center, University of Cincinnati, Cincinnati, Ohio 45229, ${ }^{3}$ Institute of Pharmacology and Toxicology, Zhejiang \\ Province Key Laboratory of Anti-Cancer Drug Research, College of Pharmaceutical Sciences, Zhejiang University, Hangzhou, China 310058, ${ }^{4}$ Institute of \\ Developmental and Regenerative Biology, Key Laboratory of Organ Development and Regeneration of Zhejiang Province, College of Life Sciences, \\ Hangzhou Normal University, Hangzhou, 310029, China, ${ }^{5}$ Department of Anatomical Sciences and Neurobiology, University of Louisville, Louisville, \\ Kentucky 40292, and ${ }^{6}$ National Centre for International Research in Cell and Gene Therapy, Centre for Cell and Gene Therapy of Academy of Medical \\ Sciences, Zhengzhou University, Zhengzhou 450052, China
}

Bergmann glia facilitate granule neuron migration during development and maintain the cerebellar organization and functional integrity. At present, molecular control of Bergmann glia specification from cerebellar radial glia is not fully understood. In this report, we show that ZEB2 (aka, SIP1 or ZFHX1B), a Mowat-Wilson syndrome-associated transcriptional regulator, is highly expressed in Bergmann glia, but hardly detectable in astrocytes in the cerebellum. The mice lacking Zeb2 in cerebellar radial glia exhibit severe deficits in Bergmann glia specification, and develop cerebellar cortical lamination dysgenesis and locomotion defects. In developing Zeb2-mutant cerebella, inward migration of granule neuron progenitors is compromised, the proliferation of glial precursors is reduced, and radial glia fail to differentiate into Bergmann glia in the Purkinje cell layer. In contrast, Zeb2 ablation in granule neuron precursors or oligodendrocyte progenitors does not affect Bergmann glia formation, despite myelination deficits caused by Zeb2 mutation in the oligodendrocyte lineage. Transcriptome profiling identified that ZEB2 regulates a set of Bergmann glia-related genes and FGF, NOTCH, and TGF $\beta / \mathrm{BMP}$ signaling pathway components. Our data reveal that ZEB2 acts as an integral regulator of Bergmann glia formation ensuring maintenance of cerebellar integrity, suggesting that ZEB2 dysfunction in Bergmann gliogenesis might contribute to motor deficits in Mowat-Wilson syndrome.

Key words: Bergmann glia; cerebellar gliogenesis and neurogenesis; cerebellar lamination; granular neuron migration; motor deficits; ZEB2

Significance Statement

Bergmann glia are essential for proper cerebellar organization and functional circuitry, however, the molecular mechanisms that control the specification of Bergmann glia remain elusive. Here, we show that transcriptional factor ZEB2 is highly expressed in mature Bergmann glia, but not in cerebellar astrocytes. The mice lacking Zeb2 in cerebellar radial glia, but not oligodendrocyte progenitors or granular neuron progenitors, exhibit severe defects in Bergmann glia formation. The orderly radial scaffolding formed by Bergmann glial fibers critical for cerebellar lamination was not established in Zeb2 mutants, displaying motor behavior deficits. This finding demonstrates a previously unrecognized critical role for ZEB2 in Bergmann glia specification, and points to an important contribution of ZEB2 dysfunction to cerebellar motor disorders in Mowat-Wilson syndrome.

\section{Introduction}

The cerebellum is essential for smoothly coordinated vestibular, motor and cognitive function. Cerebellar malformation disrupts

\footnotetext{
Received Sept. 13, 2017; revised Dec. 25, 2017; accepted Jan. 5, 2018.

Author contributions: L.H., D.M., M.X., and Q.R.L. designed research; L.H., K.Y., F.L., J.W., L.N.W., and C.Z. performed research; F.L., C.Z., Q.L., X.Z., H.L., and M.Q. contributed unpublished reagents/analytic tools; K.Y., J.W., L.N.W., Q.L., X.Z., H.L., D.M., M.Q., and Q.R.L. analyzed data; M.X. and Q.R.L. wrote the paper.

This work was funded in part by Grants from the U.S. National Institutes of Health R01NS072427 and R01NS075243 to Q.R.L., and the National Multiple Sclerosis Society (NMSS-4727) to Q.R.L. and the National Natural Science Foundation of China (81630038) to DM. We thank Guojiao Huang and Lingli Xu for technical support, Dr. Edward Hurlock for comments, and Dr. Danny Huylebroeck for Zeb2 floxed mice.
}

not only balance and locomotion (Ito, 2006), but also sensorymotor learning, speech, and spatial memory (Boyden et al., 2004; Buckner, 2013). The cerebellar cortex consists of three layers: an outer molecular layer (ML), the middle Purkinje cell layer (PCL),

\footnotetext{
The authors declare no competing financial interests.

* L.H. and K.Y. contributed equally to this work.

Correspondence should be addressed to Dr. Q. Richard Lu, Division of Experimental Hematology and Cancer Biology, Cincinnati Children's Hospital Medical Center, 3333 Burnet Avenue, Cincinnati, OH 45229. E-mail: richard.lu@echmc.org.

DOI:10.1523/JNEUROSCI.2674-17.2018

Copyright $\odot 2018$ the authors $\quad 0270-6474 / 18 / 381575-13 \$ 15.00 / 0$
} 
and an inner granular layer (IGL). The IGL neurons, the most prevalent cerebellar neurons, are derived from granule neuron precursors (GNPs), which are peripherally located in the external germinal layer (EGL) during early postnatal stages. The PCL contains the soma of Purkinje neurons and Bergmann glia.

Bergmann glia are "specialized" radial astrocytes in the cerebellar cortex, where their cell bodies are located in the PCL and processes extend into the ML layer, terminating at the pial surface (Hatten, 1999; Wang and Zoghbi, 2001). During early postnatal stages, GNPs proliferate in the EGL and migrate radially along the Bergmann glial process in an outside-in manner to form mature IGL neurons in the cerebellum (Sillitoe and Joyner, 2007; Millen and Gleeson, 2008). Bergmann glia also interact with Purkinje neurons and facilitate synaptic transmission and maintenance (Bellamy, 2006; López-Bayghen et al., 2007). Impairment of Bergmann-gliamediated GNP migration disrupts the laminar structure of the cerebellum and synaptic connections and thereby disrupts cerebellar functions (Roussel and Hatten, 2011; Buckner, 2013).

Bergmann glia are derived from radial glia in the cerebellar ventricular zone through retraction of apical processes (Yuasa, 1996; Yamada and Watanabe, 2002). During the radial-toBergmann glial transition, which occurs between mouse embryonic days (E) 14.5 and E18.5, Bergmann glia precursors maintain the radial basal processes and relocate their soma from the ventricular zone to the future PCL, and subsequently differentiate into mature Bergmann glia at $\sim$ P6 (Yuasa, 1996; Yamada and Watanabe, 2002). Bergmann glial radial fibers facilitate inward migration of differentiating GNPs from the EGL to form the IGL (Buckner, 2013).

Coordinated spatial and temporal gene expression drives the production and patterning of Bergmann glia, which are required for proper cerebellum lamination (Sudarov and Joyner, 2007). A number of signaling pathways have been implicated in regulating these processes. FGFR signaling in Bergmann glia is required for normal cerebellar architecture formation (Müller Smith et al., 2012). In addition, Notch signaling ligand DNER and NOTCH1/ RBP-J are critical for monolayer formation and morphogenesis of Bergmann glia (Lütolf et al., 2002; Eiraku et al., 2005; Komine et al., 2007). Integrin-linked kinase signaling is also required for Bergmann glial differentiation (Belvindrah et al., 2006). Recently, a role of $P t p n 11 / S h p 2$ and ErbB3 signaling in Bergmann glia maturation was identified during the initiation of cerebellar foliation (Li et al., 2014; Sathyamurthy et al., 2015). In contrast to the multiple signaling pathways identified for Bergmann glia development, the cell-intrinsic transcriptional regulators that control Bergmann glia formation have not been fully defined.

Patients with Mowat-Wilson syndrome (MOWS), an autosomal dominant disorder, exhibit congenital developmental abnormalities such as mental retardation, growth delay, epilepsy, and motor deficits (Mowat et al., 2003). MOWS is caused by mutations in ZEB2 (Cacheux et al., 2001; Wakamatsu et al., 2001). Recent studies indicate that Zeb2-mediated gene programs control neurogenesis, the fate switch between cortical and striatal interneurons, and cortical interneuron migration in the developing brain (Seuntjens et al., 2009; McKinsey et al., 2013; van den Berghe et al., 2013; Parthasarathy et al., 2014). ZEB2, also known as SIP1 (Smad-interacting protein 1), can interact with Smads and modulate TGF $\beta / \mathrm{BMP}$ signaling to regulate oligodendroglial differentiation (Verschueren et al., 1999; Weng et al., 2012).

In this study, we find that ZEB2 is expressed in Bergmann glia and is required for Bergmann glial specification from radial glia. Mice lacking Zeb2 in radial glia failed to form Bergmann glia, and the orderly radial scaffolding formed by Bergmann glial fibers critical for cerebellar lamination was not established. Thus, our data reveal that ZEB2 is a key regulator for Bergmann glial formation during cerebellar development.

\section{Materials and Methods}

Animals. Zeb2 $2^{\text {lox/lox }}$ mice (Wu et al., 2016) were crossed with hGFAP-Cre or Atoh1/Math1-Cre (He et al., 2014), or Olig1-Cre (Xin et al., 2005) mice to produce control $\left(Z e b 2^{\text {lox/+ }}: \mathrm{Cre}^{+/-}\right)$and Zeb2 conditional knock-out

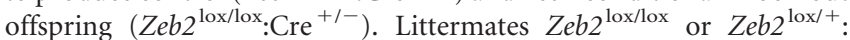
$\mathrm{Cre}^{+/-}$mice were used as controls. Transgenic hGFAP-Cre and GFAP-GFP reporter mice were obtained from The Jackson Laboratory, FVB-Tg(GFAP-cre)25Mes/J and FVB/N-Tg(GFAPGFP)14Mes/J, respectively. Animals of either sex were used in the study and littermates were used as controls unless otherwise indicated. The GFAP-GFP line was intercrossed with the hGFAP-Cre line to obtain Zeb2-conditional knock-out (cKO) mice carrying the GFAP-GFP transgene. The mouse strains used in this study were generated and maintained on a mixed C57BL/6;129Sv background and housed in a vivarium with a $12 \mathrm{~h}$ light/ dark cycle. All animal experiments were conducted in mice of both genders. All animal use and studies were approved by the Institutional Animal Care and Use Committee at the Sichuan University and the Cincinnati Children's Hospital Medical Center.

Immunostaining and in situ hybridization. The brains of mice at defined ages were dissected and fixed for $45 \mathrm{~min}$ in $4 \%$ PFA, embedded in $4 \%$ agarose, and sectioned at $50 \mu \mathrm{m}$ (for postnatal samples) and $60 \mu \mathrm{m}$ (for embryonic samples) as longitudinal vibratome-sections. For BrdU pulse labeling, animals were injected subcutaneously with $100 \mathrm{mg}$ $\mathrm{BrdU} / \mathrm{kg}$ body weight for appropriate times before collection. For immunostaining, we used antibodies to ZEB2 (rabbit; Santa Cruz Biotechnology, SC-48789), BLBP (rabbit; Abcam, ab32423), ZIC1 (rabbit; Rockland, 200-401-159), Calbindin (rabbit; Immunostar, 24427), NeuN (mouse; Millipore, MAB377), GFAP (mouse; Sigma-Aldrich, G3893), MBP (goat; Santa Cruz Biotechnology, SC-13914), Ki67 (rabbit; Thermo Scientific, RM-9106), and BrdU (rat; Abcam, ab6326). Secondary antibodies conjugated to Cy2, Cy3, or Cy5 were from Jackson ImmunoResearch Laboratories. All images were acquired using an Olympus Fluoview FV1000 confocal microscope and quantified in a doubleblinded manner by ImageJ (https://imagej.nih.gov/ij/). Images from at least five sections per animal were collected for analysis. RNA in situ hybridization was performed using digoxigenin-labeled riboprobes as described previously. The probes used were as follows: murine Glast/ Slc1a3, Fgfr2, Ntng2, Gdf10, Notch2, and Hes5. Detailed protocols are available upon request. Images were taken using a Nikon Eclipse 80i microscope.

Cell number quantification in the cerebellum. Cerebella were sectioned sagittally at $50 \mu \mathrm{m}$. Cerebellar hemispheric sections were immunostained with cell-type-specific markers and counted by visualizing labeling positive cells in the parasagittal sections using ImageJ software. Three individual mice from each group were used for the experiment, and at least four sagittal sections from each animal were used to count the total number of labeled cells in a genotyping blinded manner. Data were analyzed with unpaired Student's $t$ tests.

RNA isolation and quantitative real-time PCR. RNAs from control and Zeb2-cKO mouse cerebella were extracted using TRIZOL (Life Technologies). cDNA was synthesized from $1 \mu \mathrm{g}$ RNA using PrimeScript RT reagent Kit (Takara) according to the manufacturer's instructions. QRTPCR was performed using the CFX96 Touch Real-Time PCR Detection System (Bio-Rad). qRT-PCR was performed using FAST qPCR Kit Master Mix (KAPA Biosystems). Primers used for qRT-PCR analysis are as follows: Zeb2: Forward, accttacgaatgcccaaact; Reverse, gggaagaacccgtcttgatatt; Glast/Slcla3: Forward, aagttcagagcctcaccaag; Reverse, ctcattttatacggtcggaggg; Fgfr2: Forward, tatggaag aggaccagggatt; Reverse, tggttctaaagtggtatcctcaac; Notch2: Forward atgaagacgaagatgctgagg; Reverse, catcagctctcgaatagcgg Hes1: Forward, ggcgaagggcaagaataaatg; Reverse, gtgcttcacagtcatttccag; Hes5: Forward, gaaacacagcaaagccttcg Reverse, agcttcatctgcgtgtcg; Gdf10: Forward, gtcctcattgccctcgg; Reverse, cggttgtacttctcatagagcc; Ntng2: Forward, gctctcccaatgcctgtg; Reverse, gttgtgtttacagctgacgc. 
Transient transfections and luciferase assays. For reporter assays, HEK293 cells were transfected with pGL3-luciferase reporters driven by indicated promoters carrying ZEB2 consensus binding sites to the transcription start site ( Fgfrl: -1.9 to $-0.1 \mathrm{~kb} ;$ Fgfr2: +1.3 to $+2.8 \mathrm{~kb}$; Hes1: -5.0 to $-2.3 \mathrm{~kb}$; Hes5: -1.5 to $+0.1 \mathrm{~kb}$; erbB3: -0.3 to $+1.2 \mathrm{~kb}$; Gdf10: -2.8 to $-1.6 \mathrm{~kb}$ ) using PolyJet per the manufacturer's protocol and assayed $48 \mathrm{~h}$ post-transfection for luciferase activity by using a Promega luciferase assay kit according to the manufacturer's instructions. The pSV- $\beta$-galactosidase control vector was included to control for variable transfection efficiencies between independent experiments.

RNA sequencing and data analysis. RNA from control and Zeb2 mutant cerebella were extracted using TRIZOL (Life Technologies) followed by purification using an RNeasy Mini Kit (Qiagen). RNA-seq libraries were prepared using Illumina RNA-Seq Preparation Kit and sequenced by HiSeq 2000 Sequencer. RNA-seq reads were mapped using TopHat with default settings (http://tophat.cbcb.umd.edu). TopHat output data were then analyzed by Cufflinks to (1) calculate FPKM values for known transcripts in mouse genome reference, and (2) test the changes of gene expression between mutant and control. GO-analysis of gene expression changes was performed using Gene Set Enrichment (GSEA; http://www. broadinstitute.org/gsea/index.jsp). Normalized enrichment score (NES) reflects the degree to which the gene-set is overrepresented at the top or bottom of a ranked list of genes. The GSEA summary plots showing upregulated and downregulated pathways were plotted according to (https://www.biostars.org/p/168044/). Genes categorized with negative or positive NES are downregulated or upregulated, respectively. Circle size is proportional to the number of significant genes defined here as the number of genes represented in the leading-edge subset, i.e., the subset of members within a gene set that shows statistically significant, concordant differences between two biological states and contribute most to the NES. Circle colors represent FDR $q$ values. The heat map was generated based on $\log 2$ [FPKM] (fragments per kilobase of transcript per million mapped reads) by AltAnalyze (http://www.altanalyze.org/) with normalization of rows relative to row mean.

Statistical analysis. All analyses were done using Microsoft Excel or GraphPad Prism 6.00 (www.graphpad.com). Data are shown in histograms as mean \pm SEM. $p<0.05$ is deemed statistically significant. Data distribution was assumed to be normal, but this was not formally tested. Count data were assumed to be nonparametric, and appropriate statistical tests were used. Statistical analysis was performed by two-tailed unpaired Student's $t$ tests. Quantifications were performed from at least three experimental groups in a blinded fashion. No statistical methods were used to predetermine sample sizes, but our sample sizes are similar to those generally used in the field. No animals or data points were excluded from analyses.

Accession code. All the RNA-seq data have been deposited in the NCBI Gene Expression Omnibus under accession number GSE84058.

\section{Results}

\section{Bergmann glia express ZEB2 in the developing cerebellar cortex}

To determine the identity of Zeb2-expressing cells in the developing cerebellar cortex, we assessed ZEB2 expression by immunohistochemistry in GFAP-promoter driven GFP (GFAP-GFP) transgenic mice. In these mice, the GFAP-GFP reporter is expressed in Bergmann glia in the PCL and in astrocytes in the white matter in the postnatal stages (Zhuo et al., 1997; Koirala and Corfas, 2010). ZEB2 expression in the PCL overlapped with GFAP-GFP (Fig. 1A), a surrogate marker for Bergmann glia within the PCL (Koirala and Corfas, 2010). Approximately 98\% and $90 \% \mathrm{ZEB}^{+}{ }^{+}$cells were colabeled with GFP in the PCL at P0 and P6, respectively (Fig. 1B). GFAP-GFP colabeling with BLBP (brain lipid-binding protein), a marker for Bergmann glia, confirmed that the GFP reporter was expressed in Bergmann glia (Fig. 1C). Consistently, at P6, ZEB2 was detected in GFAPexpressing Bergmann glia in the PCL (Fig. 1D). In contrast, ZEB2 was hardly detectable in the GFAP-GFP ${ }^{+}$(Fig. $\left.1 E, F\right)$ or GFAP ${ }^{+}$
(Fig. $1 G$ ) astrocytes of the cerebellar white matter, suggesting that ZEB2 is mainly expressed in Bergmann glia but not astrocytes in the cerebellum. We did not detect ZEB2 expression in IGL neurons marked NeuN at P6 (Fig. $1 H$ ). Similarly, ZEB2 was essentially absent in the granule neuron progenitors in the EGL at P0 (Fig. 1I). In the developing cerebellum, a population of ZEB2 was detected in $\mathrm{SOX}_{2}{ }^{+}$radial glia or neural stem/progenitors in the ventricular zone (VZ) of the cerebellum at E14.5 (Fig. 1J). These observations suggest that ZEB2 expression is detected in the radial glia and Bergmann glia in the developing cerebellum.

\section{Deletion of Zeb2 in radial glia/neural progenitors disrupts cerebellar development}

To assess the function of Zeb2 in Bergmann glia formation from radial glia/neural progenitors during cerebellar development, we bred Zeb2-floxed mice with a human GFAP promoter driven-Cre (hGFAP-Cre) deleter line (Zhuo et al., 2001). hGFAP-Cre is expressed in cerebellar radial glia or neural stem/progenitors, which can give rise to the majority of the cell types including Bergmann glia, astrocytes, oligodendrocytes, and granule neurons in the cerebellum (Zhuo et al., 2001; Malatesta et al., 2003; Yue et al., 2006). The resulting Zeb2-ablated mice ( $h G F A P-C r e-Z e b 2^{f l f f l}$, referred to here as Zeb2-cKO mice) were born at a normal Mendelian ratio; however, Zeb2-cKO mice developed severe tremors and defects in balance control beginning $\sim 2$ weeks after birth. The phenotypes of Zeb2 heterozygous control animals $\left(Z e b 2^{f l /+}\right.$ : $h G F A P-C r e)$ are essentially the same as those in wild-type or $Z e b 2^{f l / f l}$ mice. In contrast to control mice, which stride forward with a regular gait in a footprint test, the Zeb2-cKO mice exhibited irregular gaits and had difficulty in maintaining equilibrium (Fig. 2A), indicating that locomotor function is severely impaired in mice that lack Zeb2.

At E18.5, multiple lobular foliations were detected in the cerebella of heterozygous control animals that were similar to that in wild-type animals. In contrast, cerebellar foliation and fissures between adjacent lobules were barely detectable in Zeb2-cKO mutants at this stage (Fig. 2B). At P28, the cerebella of control mice exhibited well organized cerebellar lobules with formation of distinct molecular layers and granular layers, whereas Zeb2cKO mice had smaller cerebella with disorganized lobular structures (Fig. 2C,D). Consistently, the cerebellum in the Zeb2-cKO mice exhibited hypoplasia in the cerebellar vermis and hemisphere (Fig. 2C), and appeared smaller and does not reach the size of the control cerebellum. In contrast to the disappearance of GNPs in EGL due to their inward migration in control mice at P28, clusters of GNPs were retained at the surface of the cerebellum in the Zeb2 mutant mice (Fig. 2E, arrows).

To determine whether ectopic cells were GNPs that had not successfully migrated, we stained control and Zeb2-cKO cerebella for ZIC1, which marks both GNPs in the EGL and mature neurons in the granular layer (Wang and Zoghbi, 2001; He et al., 2014), and for NeuN, which marks mature neurons in the IGL. In control mice at P18, the majority of GNPs had migrated to the IGL (Fig. $2 F$ ). Only a few $\mathrm{ZICl}^{+}$cells were present in the ML; these cells are likely completing the migration process from EGL to the IGL. In contrast, clusters of $\mathrm{ZICl}^{+}$and $\mathrm{NeuN}^{+}$neurons were detected in the EGL and ML in Zeb2-cKO cerebella (Fig. 2E, inset). The ectopic cell clusters stayed on the surface of ML in the cerebellar cortex, however, they were not Ki $67^{+}$proliferative GNPs (Fig. $2 G$ ), suggesting that they are postmitotic GNPs or immature granule neurons, and fail to migrate into the molecular layer to become mature granule neurons in the IGL of the Zeb2-cKO mice. 
A
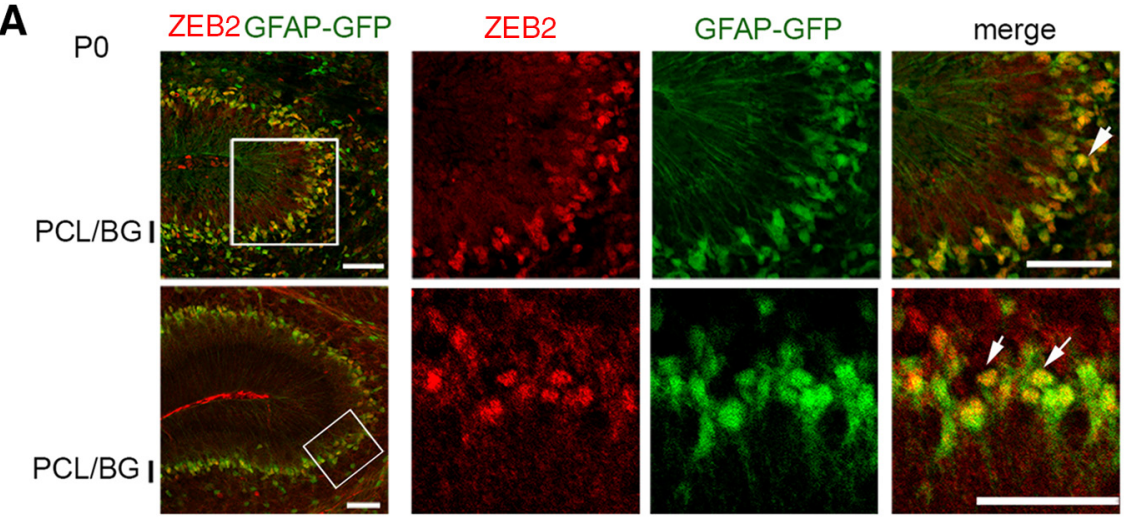

B
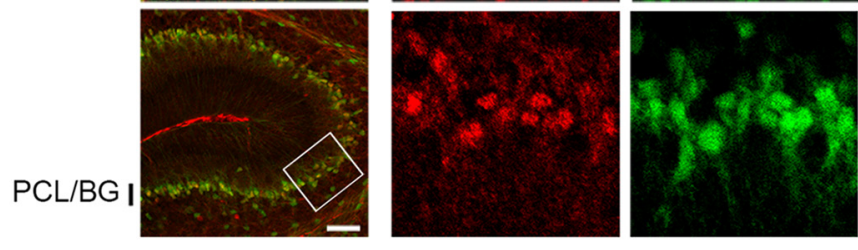

D

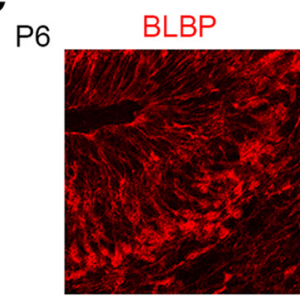

GFAP-GFP BLBPGFAP-GFP
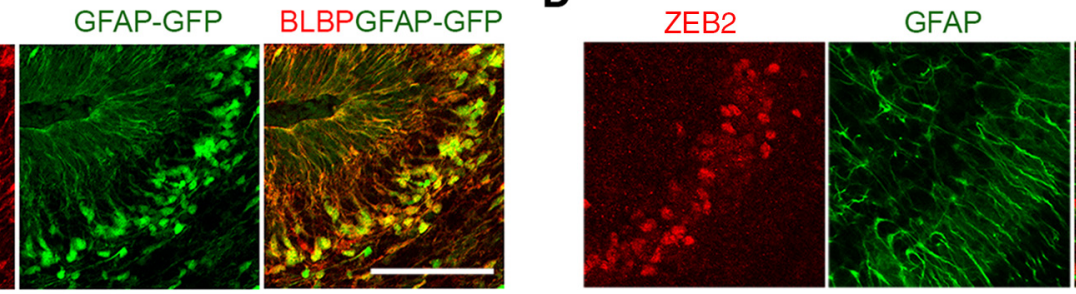

merge

E

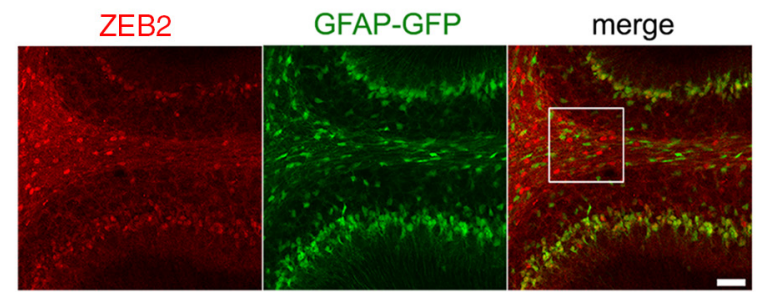

G
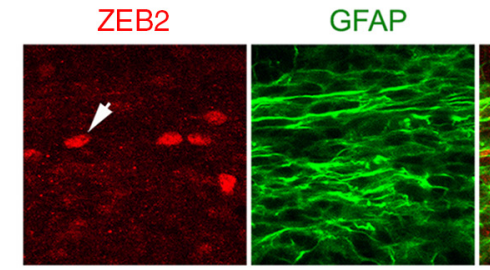

merge

F
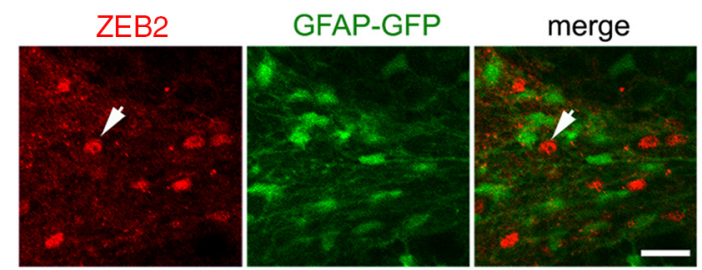

I PO
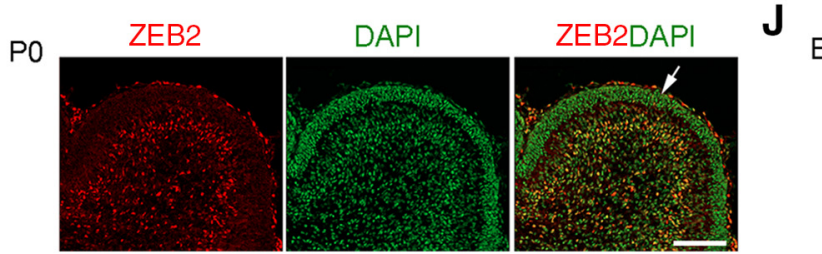

H

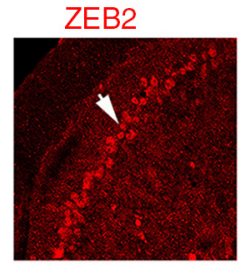

NeuN
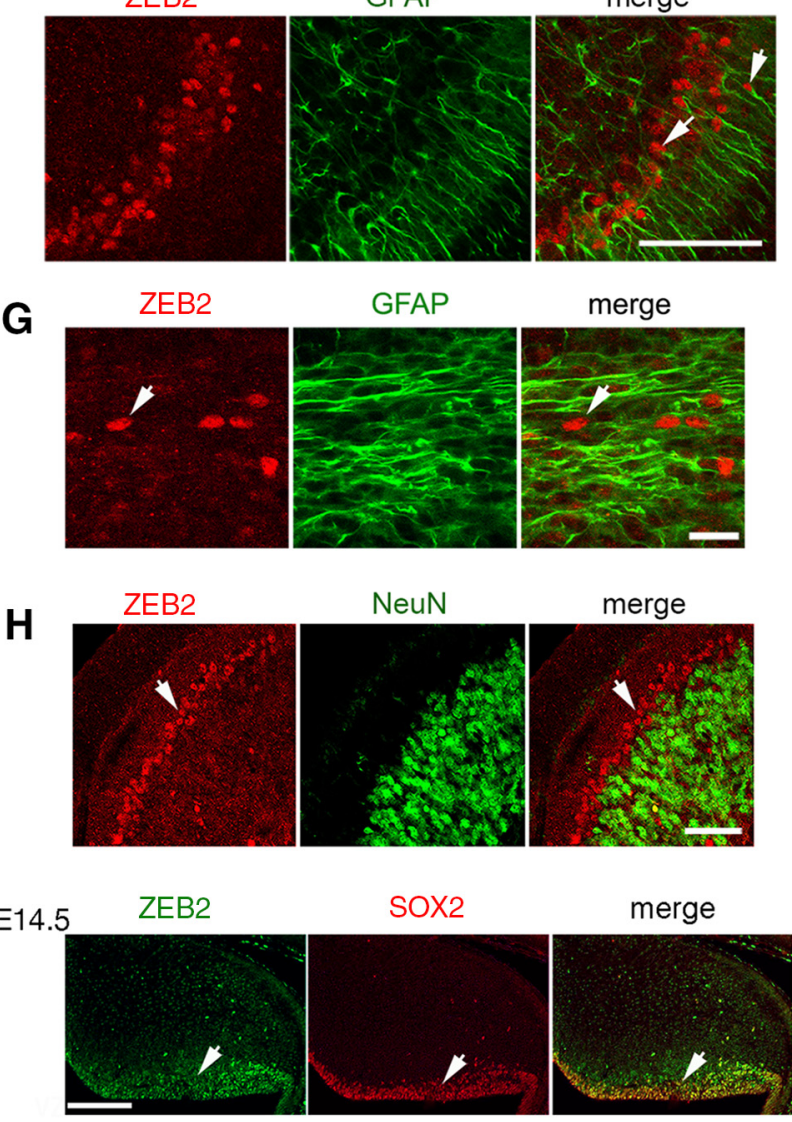

Figure 1. ZEB2 expression is restricted to cerebellar Bergmann glia. $A$, Immunostaining for ZEB2 in sagittal cerebellar sections of GFAP-GFP transgenic mice at P0 and P6. The white-boxed regions on the left are shown at higher-magnification on the right. Arrows indicate co-labeling cells. Scale bars, $100 \mu \mathrm{m}$. $\boldsymbol{B}$, Bar graph showing the percentage of ZEB2 ${ }^{+}$and ZEB2 ${ }^{-}$cells among GFAP-GFP ${ }^{+}$cells at P0 and P6 in wild-type mice ( $n=4$ animals/stage). C, Immunostaining for BLBP in sagittal cerebellar sections of GFAP-GFP transgenic mice at P6. Scale bar, $100 \mu$ m. $D$, Double-immunostaining for ZEB2 with GFAP in the WT cerebellum at P6. Arrows indicate co-labeling cells. Scale bars, $100 \mu \mathrm{m}$. E, Immunostaining for ZEB2 in sagittal cerebellar sections of GFAP-GFP transgenic mice at P6. Scale bars, $25 \mu \mathrm{m}$. $\boldsymbol{F}$, High-magnification image of the boxed region in E. Scale bars, $25 \mu \mathrm{m}$. G, Double-immunostaining for ZEB2 with GFAP in the WT cerebellum at P6. Scare bars, $25 \mu \mathrm{m}$. $\boldsymbol{H}$, Double-immunostaining for ZEB2 with NeuN in the WT cerebellum at P6. Scale bars, $100 \mu \mathrm{m}$. I, Immunostaining of ZEB2 and DAPI (4', 6-diamidino-2-phenylindole) staining for the EGL in cerebella of wild-type mouse at P0. Scale bars, $100 \mu \mathrm{m}$.J, Double staining for ZEB2 and SOX2 in the cerebellum at E14.5. Arrows indicate SOX2/ZEB2 co-labeling positive cells in the VZ. Scale bars, $50 \mu \mathrm{m}$.

Impaired radial migration of granule neurons in Zeb2-cKO mutants A characteristic feature of Bergmann glia is that they exhibit unipolar morphology and form parallel processes that provide scaffolding for GNP migration during cerebellar development. Beginning at P6, GNPs begin to migrate inward along Bergmann glia processes and eventually cross the PCL to form the IGL. In control mice at $\mathrm{P} 10, \mathrm{GFAP}^{+}$Bergmann glial processes were parallel to each other and projected into the pial surface along the ML (Fig.
$3 A$ ). These migrating GNPs were visible in the ML as were GNPs distributed along the EGL in wild-type cerebella (Fig. 3A). Postmitotic granule neurons, which have successfully migrated across the PCL, form the IGL. Cerebellar cortical lamination was distinctly organized into the EGL, ML, and IGL at P10 (Fig. 3A).

In contrast to control mice, no parallel radial processes were detected in Zeb2-cKO mutants due to the absence of Bergmann glia at P10 (Fig. $3 A$ ). In addition, GFAP-GFP ${ }^{+}$cells were scat- 
A

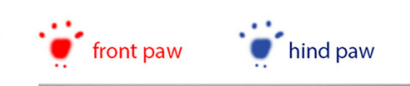

B

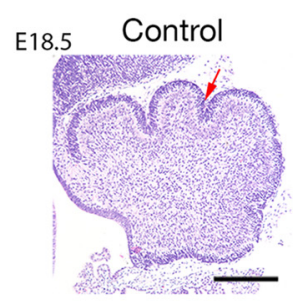

C

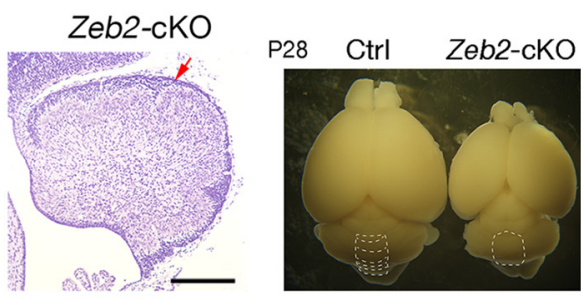

E

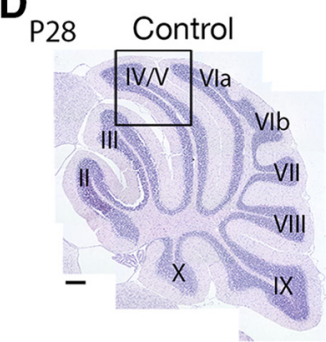

Zeb2-cKO-1

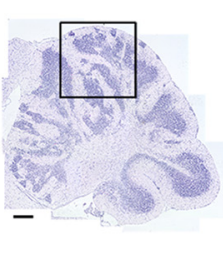

Zeb2-cKO-2
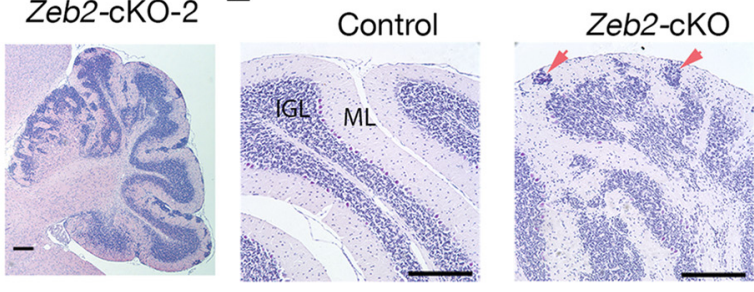

G

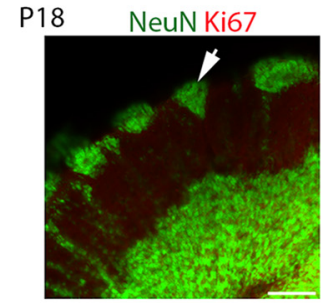

Figure 2. ZEB2 is required for normal cerebellar development. $\boldsymbol{A}$, Footprint test in the control (Zeb2 $2^{f /+}: h$ GFAP-Cre) and Zeb2-cK0 mice at P20. B, Hematoxylin and eosin (H\&E) stain shows the morphology of the control and Zeb2-cKO cerebella at E18.5. Arrows indicate primordial fissures. Scale bars, $100 \mu \mathrm{m}$. C, Whole-mount view of brains of control (Zeb2 ${ }^{f / /+}$ : hGFAP-Cre) or Zeb2-cK0 mutant mice at P28. The white dashed line indicates the cerebellar vermis. $\boldsymbol{D}, \boldsymbol{E}$, H\&E stain showing the lobule formation of the cerebellum in control and two individual Zeb2-cK0 mice at P28. Lobules are labeled with Roman numerals. The boxed regions in $\boldsymbol{C}$ are shown respectively at higher-magnification in $\boldsymbol{D}$. Arrows indicate the ectopic cell mass. Scale bars, $100 \mu \mathrm{m}$. F, Double-staining for ZIC1 and NeuN in control and Zeb2-cKO mice at P18. Insets, Arrowhead and arrow indicate EGL and the ectopic cell mass in the ML, respectively. Scale bars: main panels, $100 \mu \mathrm{m}$; insets, $20 \mu \mathrm{m}$. G, Immunostaining for Ki67 and NeuN in the Zeb2-cK0 cerebellum at P18. Scale bars, $100 \mu \mathrm{m}$.

tered across the ML/PCL of Zeb2-cKO mutants compared with those lining along the PCL in the control (Fig. 3B). Furthermore, the number of Bergmann glia-like cells $\left(\mathrm{GFP}^{+}\right.$cells with radial processes) were much reduced in Zeb2-cKO animals at $\mathrm{P} 3$ and P10 (Fig. 3C). The majority of GFAP-GFP ${ }^{+}$glia detected in the Zeb2-cKO cerebellar cortex expressed astrocytic S100 $\beta$ (Fig. 3D) without unipolar processes, suggesting that these GFAP-GFP ${ }^{+}$/ $S 100 \beta^{+}$cells represent astrocytes. In the mutant cerebella, $\mathrm{ZICl}^{+}$granule neurons or their progenitors were dispersed throughout the EGL, ML, and IGL without a clear ML boundary between EGL and IGL as in the control (Fig. $3 A$ ).

To verify GNP migration defects, we performed BrdU fatetracing study of proliferating GNPs by injecting BrdU at P6, when the majority of GNPs are proliferative, into control and mutant mice. The cerebella were harvested $110 \mathrm{~h}$ after BrdU injection at P6 and immunostained for BrdU and calbindin. In control mice, we observed very few granule neurons with BrdU label in the ML. Most BrdU label-retaining GNPs had migrated through PCL, marked by calbindin labeling, into the IGL (Fig. 3E). In the Zeb2-cKO cerebella, however, the majority of the BrdU-labeled GNPs remained in the EGL and ML, and very few $\mathrm{BrdU}^{+}$GNPs migrated into the IGL (Fig. 3E, F). These observations suggest that deletion of Zeb2 in radial glia blocks Bergmann glial formation, leading to defects in GNP migration into the IGL.

\section{Specification of Bergmann glia is impaired in Zeb2-cKO mutants}

In control embryos, at E15.5, GFAP-GFP-expressing precursors appear to migrate outward to the cerebellar cortex from the ventricular zone with long radial processes directed toward the pial surface (Fig. 4A). By E18.5, the GFAP-GFP ${ }^{+}$Bergmann glia had successfully reached their destination and cell bodies were aligned with the PCL (Fig. 4B). In contrast, in Zeb2-cKO embryos, the GFAP-GFP ${ }^{+}$cells were barely detectable in the cerebellar cortex at E15.5. At E18.5, we however did not detect these $\mathrm{GFAP} \mathrm{GFP}^{+}$glial cells in the location along the PCL occupied principally by Bergmann glia (Fig. $4 B$ ). Most of the detectable GFAP-GFP-expressing glia were dispersed throughout the inner cerebellar region in Zeb2-cKO mutants. These observations suggest a deficit in the specification of Bergmann glia in the developing cerebellum of Zeb2-cKO mutants. There were significantly fewer $\mathrm{GFP}^{+}$glia in the presumptive PCL in Zeb2cKO animals than in controls at E18.5 (Fig. 4C). The number of activated cleaved-caspase 3-labeled cells in the cerebellum was similar between control and Zeb2 mutant animals (data 
A

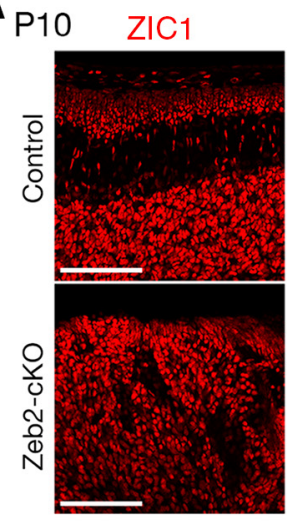

GFAP ZIC1GFAPDAPI

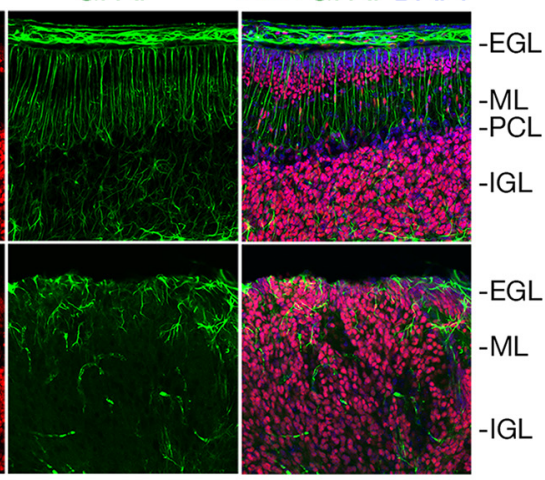

B
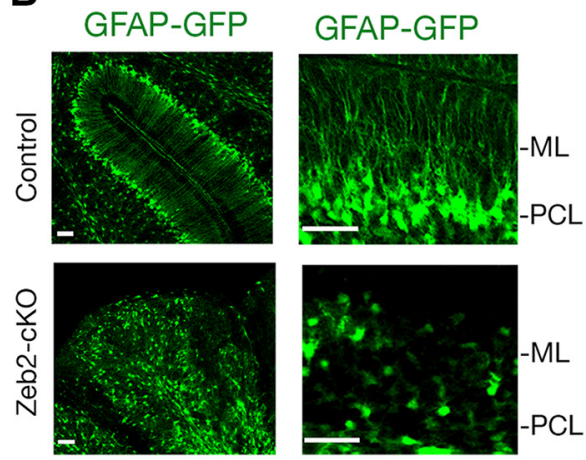

D

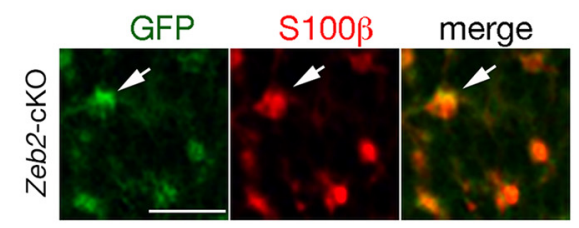

$\mathbf{F}$

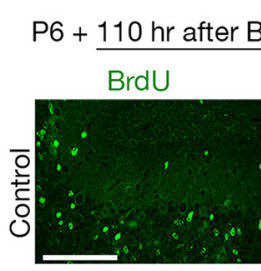

P3
- Control

= Zeb2-cKO

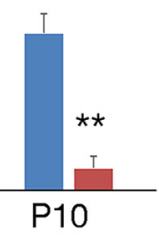

\section{E}

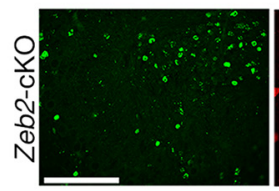

CalbindinBrdUDAPI
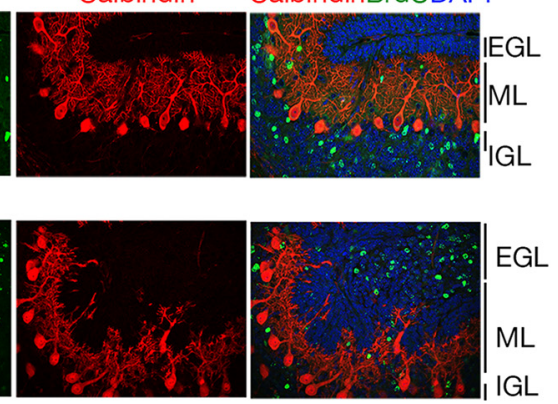

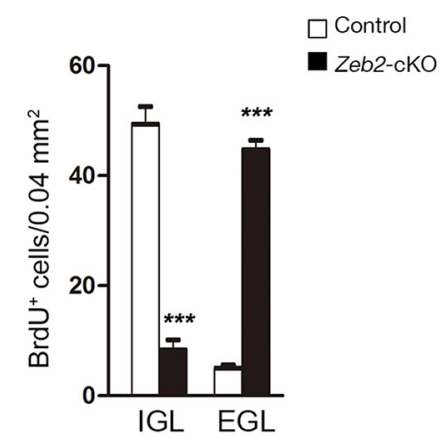

Figure 3. Migration defects of granule neurons in Zeb2-deficient cerebella. A, Double-staining for ZIC1 and GFAP in cerebella of control and Zeb2-cK0 mice at P10. Scale bars, $100 \mu \mathrm{m}$. $B$, GFAP-GFP expression in the ML of control and Zeb2-cK0 cerebella at P10. Right, High-magnification of the PCL layer. Scale bars, $50 \mu \mathrm{m}$. C, Quantification of the number of Bergmann glia-like cells with radial processes in the anterior lobules (II-VI) from control and Zeb2-cKO cerebella at P3 and P10 ( $n=3$ animals/genotype/time-point). Data represent means \pm SEM. ${ }^{* *} p<0.01$, Student's $t$ test. $\boldsymbol{D}$, Immunostaining for $S 100 \beta$ in Zeb2-cK0 mutant cerebellum carrying the GFAP-GFP transgene at P10. Arrows indicate the S100 $\beta /$ GFP double-positive cells. Scale bars, $30 \mu \mathrm{m}$. E, Double-staining for BrdU and calbindin in cerebella of control and Zeb2-ck0 mice at P10 after BrdU labeling for $110 \mathrm{~h}$ beginning at P6. Scale bars, $100 \mu \mathrm{m}$. $F$, Quantification of the number of BrdU ${ }^{+}$cells in indicated layers per area $\left(0.1 \mathrm{~mm}^{2}\right)$ in $B\left(n=3\right.$ animals/genotype). Data represent means \pm SEM. ${ }^{* * *} p<0.001$, Student's $t$ test.

not shown), suggesting that the reduction in the number of Bergmann glia is not due the compromised cell survival.

To evaluate the effects of ZEB2 on the proliferation of GFAP$\mathrm{GFP}^{+}$glia, we immunostained for a cell proliferation marker Ki67 in cerebella during embryogenesis, when Bergmann glia undergo extensive proliferation. At E15.5 and E17.5, the number of $\mathrm{GFP}^{+}$cells stained for Ki67 in the presumptive PCL was reduced in Zeb2-cKO animals (Fig. 4D-F). Similarly, the proliferation rate of GFAP-GFP ${ }^{+}$cells (the percentage of $\mathrm{Ki}^{+} 7^{+} \mathrm{GFP}^{+}$ among $\mathrm{GFP}^{+}$cells) was lower in Zeb2-cKO cerebella compared with controls (Fig. $4 G$ ), suggesting that the defects in Bergmann glia generation is in part attributed to the deficiency of GFAP$\mathrm{GFP}^{+}$glial precursor expansion in Zeb2-cKO mutants.

Granule neurons and Purkinje cells are generated in the Zeb2-cKO cerebellum

To determine whether the formation of GNPs in the EGL was affected due to the loss of Zeb2 in cerebellar neural progenitors, we quantified GNPs in the EGL in control and Zeb2-cKO mutant cerebella assayed by expression of ZIC1, which marks both outer EGL (mitotic GNPs) and the inner EGL (postmitotic GNPs; Wang and Zoghbi, 2001). At P3, the density of $\mathrm{ZIC1}^{+}$GNPs in the EGL was comparable between control and Zeb2-mutants (Fig. $5 A, B$ ). In addition, we did not detect a significant difference in the percentage of $\mathrm{BrdU}^{+}$GNPs in the EGL between control and Zeb2-cKO mice (Fig. 5C,D), suggesting that Zeb2 deletion in radial glia does not affect GNP generation.

To determine the effects of Zeb2 ablation on Purkinje cell development, we examined expression of calbindin, a Purkinje cell marker, at postnatal stages. Calbindin ${ }^{+}$Purkinje cells aligned to form a monolayer and extended their dendrites toward the EGL in the control animals at P3 and P10 (Fig. 5E). In Zeb2-cKO mutants, Purkinje cells had extended dendrites, but their cell bodies aggregated into multicellular clusters (Fig. 5E). Nonetheless, overall Purkinje cell numbers were comparable between con- 
A $\mathrm{E} 15.5$

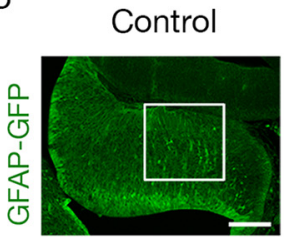

B

E18.5

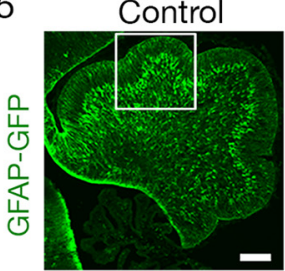

D
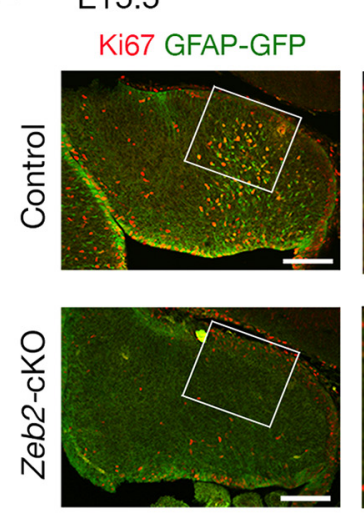

Ki67 GFAP-GFP
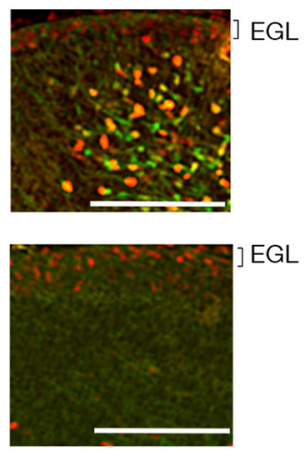

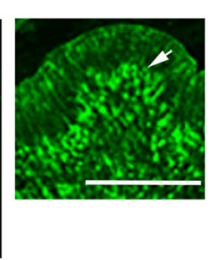

E

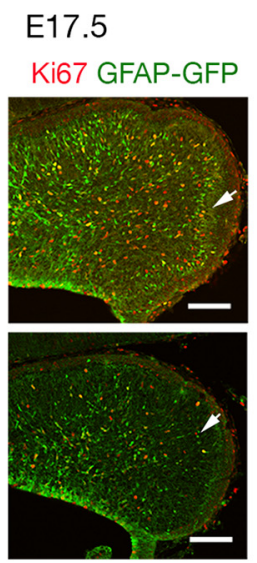

Zeb2-cKO
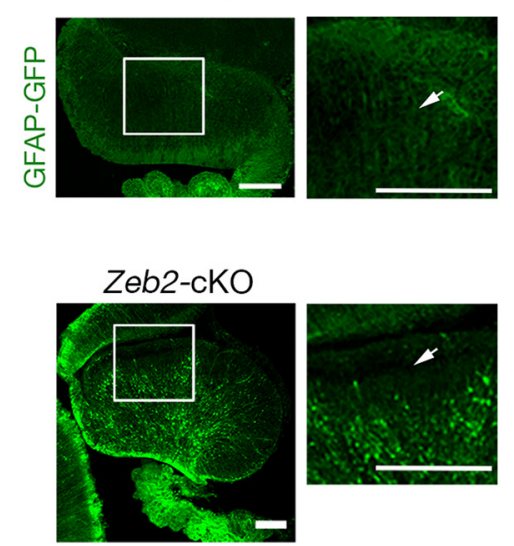

F

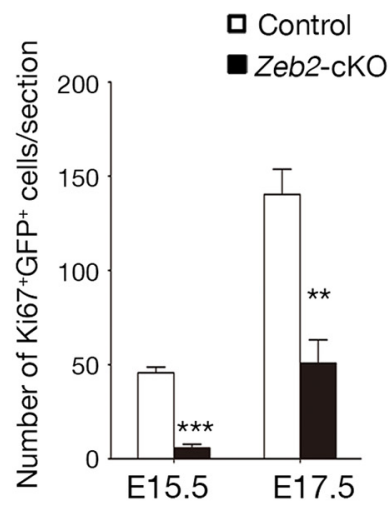

口 Control

Zeb2-cKO

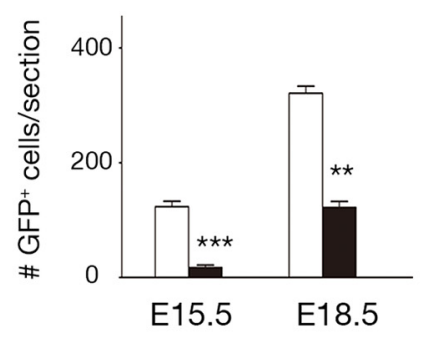

G

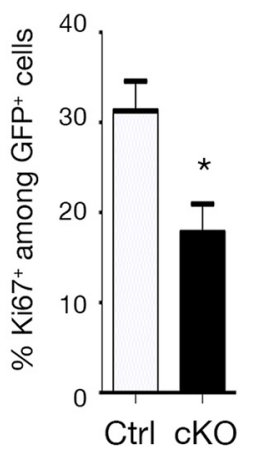

Figure 4. Bergmann glial defects in Zeb2-cK0 mice. A, B, GFAP-GFP-expressing Bergmann glial cells in control and Zeb2-cK0 cerebella at E15.5 and E18.5 as indicated. The boxed regions on the left are shown, respectively, at higher-magnification on the right. Scale bars: $A, 100 \mu \mathrm{m} ; \boldsymbol{B}, 50 \mu \mathrm{m}$. C, Quantification of GFP ${ }^{+}$cells per parasagittal section of control and Zeb2-cK0 cerebella at indicated stages ( $n=3$ animals/genotype). Data represent means \pm SEM. ${ }^{* *} p<0.01,{ }^{* * *} p<0.001$, Student's $t$ test. D, E, Immunostaining for Ki67 in control and Zeb2-cK0 cerebella of GFAP-GFP transgenic animals at E15.5 and E17.5. D, The boxed regions on the left are shown, respectively, at higher-magnification on the right. Arrows in $E$ indicate the presumptive PCL. Scale bars, $100 \mu \mathrm{m}$. $\boldsymbol{F}$, Quantification of Ki67/GFP double-positive cells in control and Zeb2-cK0 cerebella $\left(0.1 \mathrm{~mm}^{2}\right)$ at E15.5 and E17.5 $\left(n=3\right.$ animals/genotype). Data are means \pm SEM. ${ }^{* *} p<0.01$, ${ }^{* * *} p<0.001$, Student's $t$ test. G, Percentage of Ki67-positive cells among GFP + cells at E17.5. ( $n=3$ animals/genotype). Data are means \pm SEM. ${ }^{*} p<0.05$, Student's $t$ test.

trol and mutant cerebella at P10 (Fig. 5F). These observations suggest that ZEB2 is not required for the formation of granule neurons or Purkinje cells.

\section{Zeb2 deletion in oligodendrocyte progenitors or GNPs does not affect Bergmann glia formation}

Previous studies showed that ZEB2 is expressed in oligodendrocyte lineage cells and is critical for oligodendrocyte differentiation (Weng et al., 2012). To evaluate whether the defects in Bergmann glia development in Zeb2-cKO animals can be caused by myelination deficiency in a non-cell autonomous manner, we examined cerebellar development in Zeb2 $2^{f l f f l}$ :Olig1-Cre mice with Zeb2 deletion in the oligodendrocyte lineage. At P14, Purkinje cells and Bergmann glia process formation marked by calbindin and GFAP, respectively, appeared normal in the Zeb2 ${ }^{f l f l}:$ Olig1Cre mutant cerebellum. The cerebellar cortical lamination and foliation were essentially intact except for the loss of expression of MBP and ZEB2 expression in the cerebellar white matter in the Zeb2 $2^{f l f l}:$ Olig1-Cre mutant mice (Fig. 6A,B), suggesting that myelination deficits in the Zeb2 $2^{\text {flffl }}$ :Olig1-Cre mutants do not affect Bergmann glia formation.

To examine the possibility that the Bergmann glia defects in Zeb2-cKO mutant mice may be caused indirectly by the loss of
ZEB2 function in GNPs, which are derived from neural progenitors, we generated mice in which Zeb2 floxed alleles were deleted using a GNP-specific Cre line, Atoh1/Math1-Cre (Lumpkin et al., 2003; He et al., 2014). The cerebellar foliation, and the morphology and number of $S 100 \beta^{+}$Bergmann glia in the cerebellum of Zeb2 $2^{f / f l}:$ Atoh1-Cre mice were comparable to the control

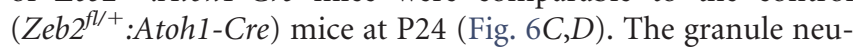
ron marker ZIC1 expression was not altered in the cerebellum of $Z e b 2^{f l / f l} ;$ Atoh1-Cre mutant mice (Fig. $6 E$ ), consistent with the lack of ZEB2 expression in GNPs at the EGL (Fig. 1). In Zeb $2^{\text {flffl }}$ : Atoh1-Cre mice at P1, BrdU-pulse labeling showed that the percentage of $\mathrm{BrdU}^{+}$cells among $\mathrm{ZICl}^{+}$GNPs in the EGL were indistinguishable from control animals (Fig. $6 F, G$ ), suggesting that deletion of Zeb2 in Atoh1 ${ }^{+}$GNPs does not affect the formation of granule neurons and Bergmann glia.

\section{Differentially expressed gene profiling in Zeb2-cKO cerebella} identifies new Bergmann glial markers

To determine the potential mechanisms underlying ZEB2 regulation of Bergmann glia development, we performed RNA-seq profiling using cerebella of control and Zeb2-cKO mice at P0, when Bergmann glial cells undergo proliferation and specification processes. The control and Zeb2-cKO groups exhibited dis- 
A

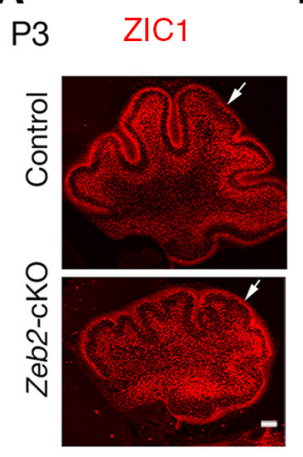

E

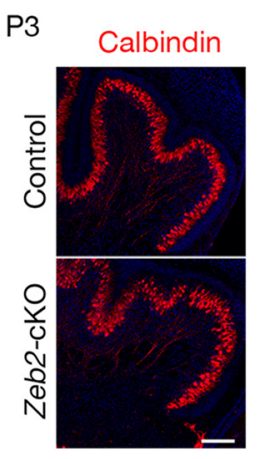

B
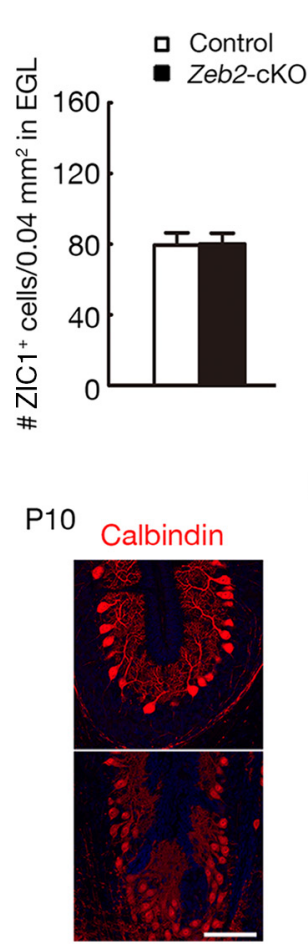

C

P3

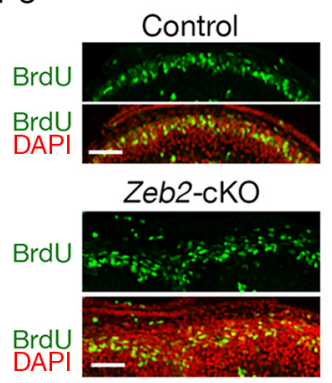

$\mathbf{F}$

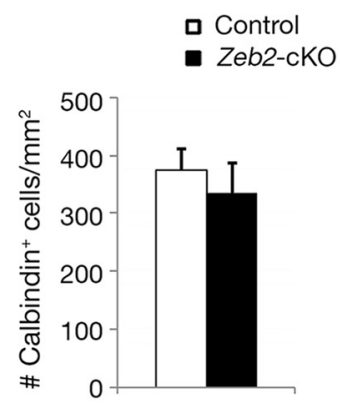

D

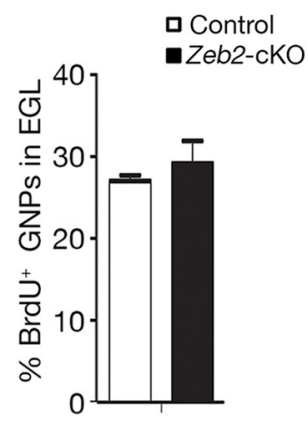

Figure 5. Granule and Purkinje cell development is normal in Zeb2-cKO mice. A, Immunostaining for ZIC1 in the of control (Zeb2 $2^{f /+}: h G F A P$-Cre) and Zeb2-cKO mice at P3. Scale bar, $50 \mu \mathrm{m}$. $\boldsymbol{B}$, The average density of ZIC1 ${ }^{+}$cells per $0.04 \mathrm{~mm}^{2}$ in the EGL of all lobules in control and Zeb2-cKO cerebella at P3 ( $n=3$ animals/genotype). Data are means \pm SEM. C, Immunostaining for BrdU and calbindin in the cerebella of control and Zeb2-cKO mice at P3 with BrdU-pulse labeled for $2 \mathrm{~h}$. Scale bars, $50 \mu \mathrm{m}$. D. Percentage of BrdU ${ }^{+}$GNPs in the EGL of control and Zeb2-cK0 cerebella at P3 ( $n=3$ animals/genotype). Data represents the mean \pm SEM. E, Immunostaining for calbindin in the cerebella of control and Zeb2-cK0 mice at P3 and P10. Scale bars, $100 \mu \mathrm{m} . \boldsymbol{F}, Q$ uantification of calbindin ${ }^{+}$cells per cerebellum section at $\mathrm{P3}$ ( $n=3$ animals/genotype). Data represents the mean \pm SEM.

tinct gene expression profiles (Fig. 7A). We identified a set of differentially expressed genes in the Zeb2-cKO cerebella relative to the control by interrogating gene expression signatures. Gene profiling analysis indicated that the genes upregulated in Zeb2cKO cerebella, were associated with epithelial-to-mesenchymal transition (EMT), which is consistent with a role of ZEB2 in EMT through repressing epithelial cell-cell junction gene expression (Vandewalle et al., 2005), GPCR signaling, calcium, STAT3 signaling, and astrocytic signature genes (Fig. 7B). Consistently, GSEA revealed an upregulation of astrocytic signatures alongside the EMT pathway in Zeb2-cKO cerebella (Fig. $7 C$ ). In contract, the genes that were downregulated in Zeb2-cKO cerebella indicated an enrichment of gene categories that are related to mitotic cell cycle, Bergmann glia- and oligodendrocyte-expressing genes (Fig. $7 B-D$ ), consistent with the defects in Bergmann glia specification and proliferation as well as myelination in Zeb2-cKO mice.

Genes known to be enriched in Bergmann glial cells like Glast $(S l c 1 \alpha 3)$ were downregulated in the Zeb2-cKO mutant (Fig. 7D). In addition, genes encoding several signaling components in the Notch pathway (e.g., Hes5 and Notch2), FGF pathway (e.g., Fgfr2, Fgf9, and Ptpn11), and the BMP pathway such as Gdf10, also known as Bmp3b, were downregulated in Zeb2-cKO cerebella. FGF/FGFR signaling (Lin et al., 2009; Müller Smith et al., 2012; Li et al., 2014), NOTCH/HES signaling (Eiraku et al., 2005; Komine et al., 2007) and BMP signaling (Mecklenburg et al., 2014) have been shown to regulate Bergmann glial development. Notably, we found that Ntng2, which encode Netrin G2 in the Netrin family functioning in axonal guidance and neuronal migration (Barallobre et al., 2005; Nishimura-
Akiyoshi et al., 2007), exhibited an expression pattern that resembles that of Bergmann glia-expressing markers like Glast and Gdf10 (Fig. 7D).

Given the defects in Bergmann glia differentiation, we focused on the downregulated genes in Zeb2-cKO mutants, which likely identified the genes that are highly expressed in Bergmann glia. To verify dysregulation in FGF, NOTCH, BMP, and Netrin pathways, we performed in situ hybridization to examine the expression of components of these pathways in control and Zeb2-cKO mutants. In control cerebella, expression patterns of Fgfr2, Hes5, Notch2, Gdf10, and Ntng2 at E17.5 were similar to that of a Bergmann glial-expressing gene Glast (Fig. 7E), suggesting that expression of these genes is enriched in Bergmann glia. In contrast, in Zeb2-cKO cerebella, expression of Fgfr2, Hes5, Notch2, Gdf10 and Ntng2 did not extend to where Bergmann glia are located along the PCL (Fig. 7E). Quantification by qRT-PCR further indicated that these mRNAs were expressed at lower levels in the Zeb2-cKO cerebella than wild-type controls (Fig. 7F), consonant with reduced in situ signals in the Bergmann glia at the PCL layer in the mutants. Consistently, Zeb2 overexpression enhanced the activity of reporter genes driven by the promoters of FGF receptors Fgfr1 and Fgfr2, a NOTCH effector Hes5, as well as a TGF $\beta$ receptor ligand $G d f 10$ (Fig. $7 G$ ), suggesting that ZEB2 expression potentially promotes FGF receptor, NOTCH and TGF $\beta$ receptor signaling pathways for Bergmann gliogenesis.

\section{Discussion}

Our studies present a previously unrecognized, essential role of the transcription factor ZEB2 for Bergmann glia specification from radial glial cells in the cerebellum. In addition to present 
A

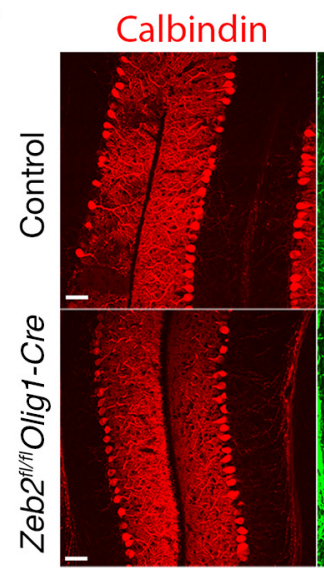

C

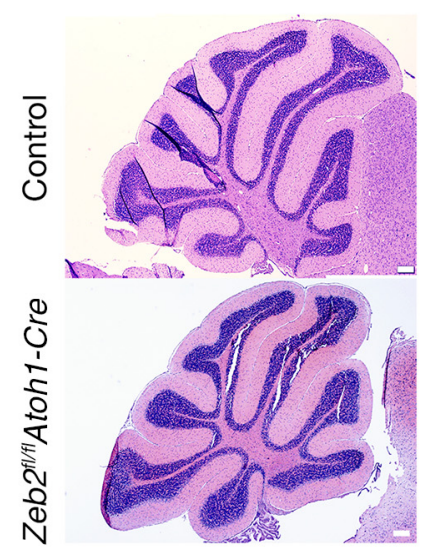

GFAP

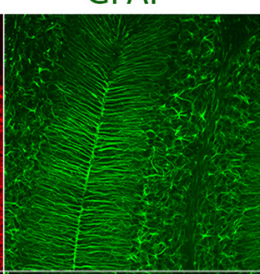

MBP

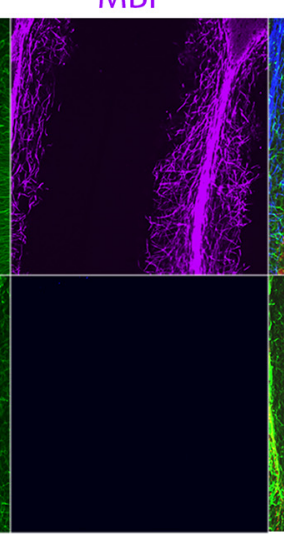

merge

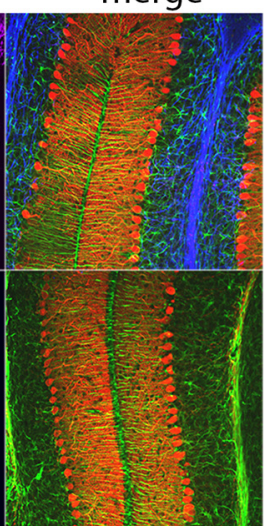

B
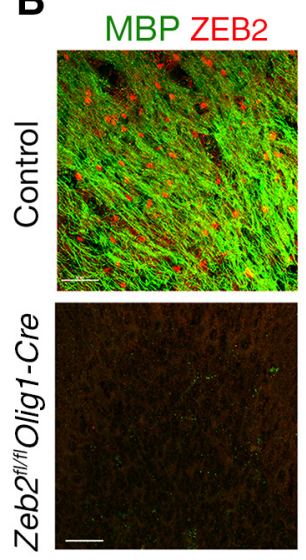

D
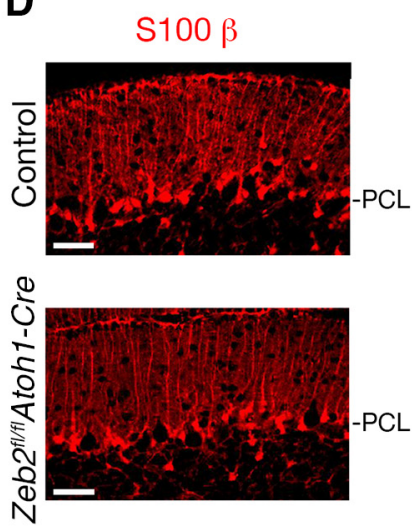

E

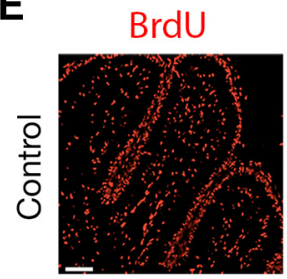

$\mathrm{ZIC1}$
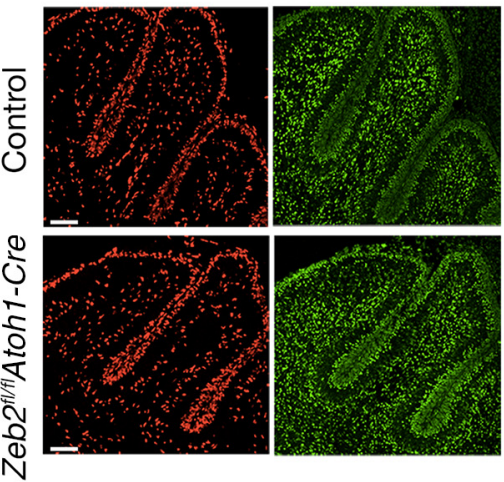

$\mathbf{F}$

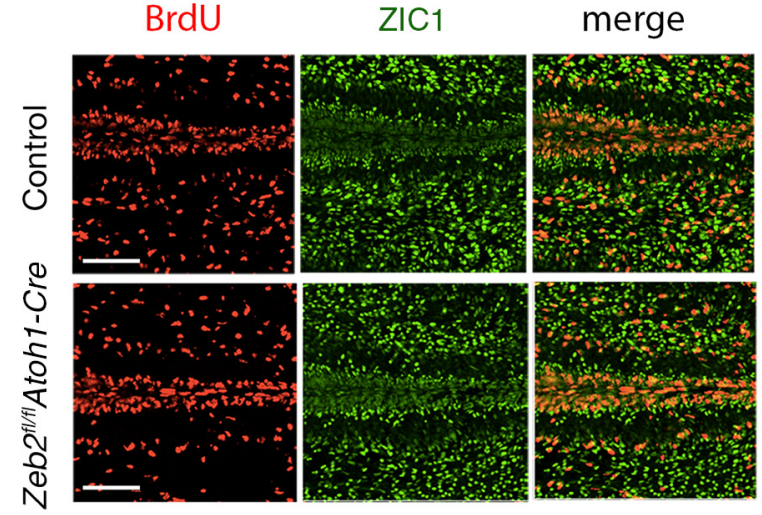

G

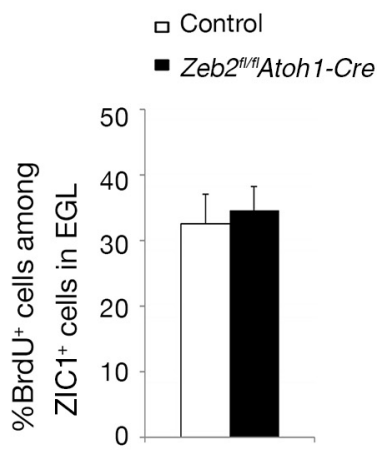

Figure 6. Zeb2 deletion in the $0 \mathrm{~L}$ lineage or GNPs does not affect cerebellar cortical development. $A$, Immunostaining for calbindin, GFAP, and MBP in cerebella of Zeb2 ${ }^{\text {fl/fl: }: 0 l i g 1-C r e ~ m o u s e ~ a n d ~}$ littermate control (Zeb2 $2^{f /+}:$ Olig1-Cre) at P14. Scale bars, $50 \mu \mathrm{m}$. B, Double immunostaining of MBP and ZEB2 in control (Zeb2 ${ }^{f / /+}:$ Olig1-Cre) and Zeb2 mutants (Zeb2 $2^{f / f f}:$ Olig1-Cre) in the oligodendrocyte lineage at P14. Scale bars, $50 \mu \mathrm{m}$. C, H\&E staining of control and Zeb2 ${ }^{f / f}:$ Atoh1-Crecerebella at P24. Scale bars, $100 \mu \mathrm{m} . D$, , S100 $\beta$ immunostaining of control and Zeb2 $2^{f / f}$ :Atoh1-Cre

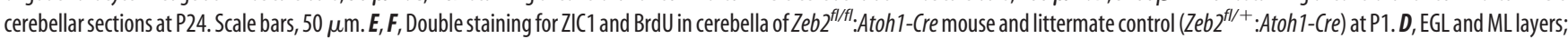
E, higher-magnification images of the interfaces between these layers. Scale bars, $100 \mu \mathrm{m}$. G, Percentage of BrdU ${ }^{+}$cells among ZIC1 ${ }^{+}$cells in the control and Zeb2 ${ }^{f / f f l}:$ Atoh 1-Cre cerebellar sections at $\mathrm{PO}(n=3$ animals/genotype). Data represent means \pm SEM.

cerebellar radial glia/neural progenitor cells in the $\mathrm{VZ}$ and oligodendrocytes, ZEB2 is highly expressed in Bergmann glia, but hardly detectable in astrocytes in the cerebellar white matter tract or interneurons in the postnatal cerebellum, consistent with expression of Zeb2/Sip1-EGFP knock-in reporter detected in Bergmann glia (Nishizaki et al., 2014). Because there are no well established specific markers to distinguish astrocytes and Bergmann glia, except for their distinct anatomical locations and morphology, ZEB2 may represent a cell nucleus-expressing marker for mature Bergmann glia that are distinct from astrocytes in the cerebellar cortex.

During embryonic development, Bergmann glia precursors are derived from the radial glial cells in the VZ. The nascent Bergmann glia precursors continue to proliferate until P7, when they exit the cell cycle and differentiate into mature Bergmann glia (Parmigiani et al., 2015). The lack of radial process-bearing Bergmann glial formation, but not astrocytes, in the presumptive PCL in the Zeb2-cKO embryos, where ZEB2 is deleted in radial 
A

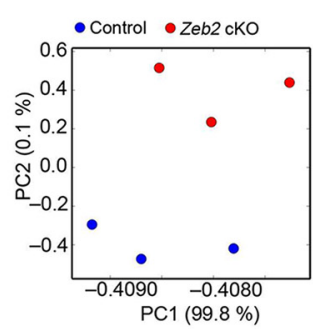

D

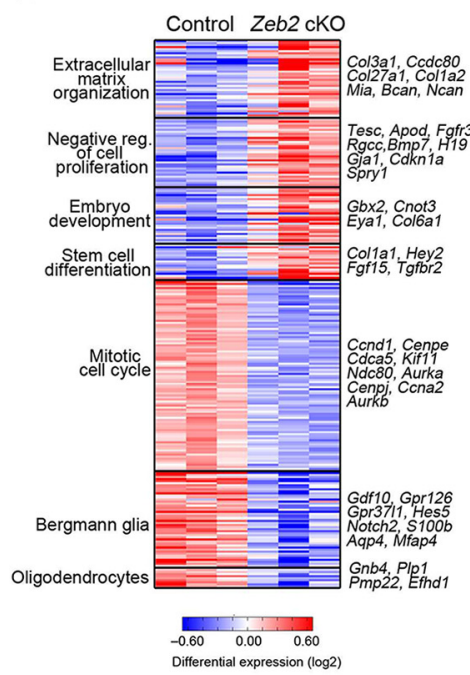

B

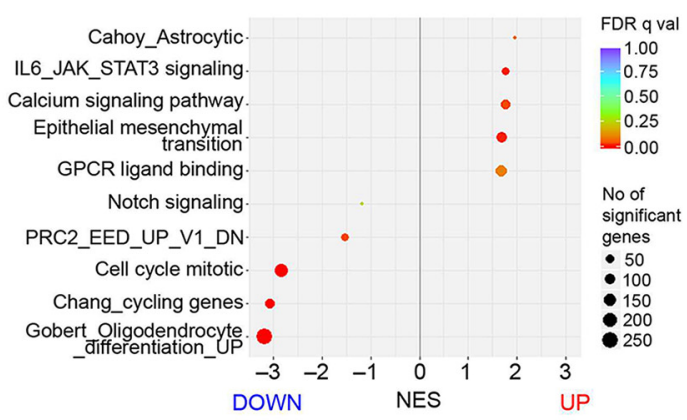

\section{E}

E17.5
C

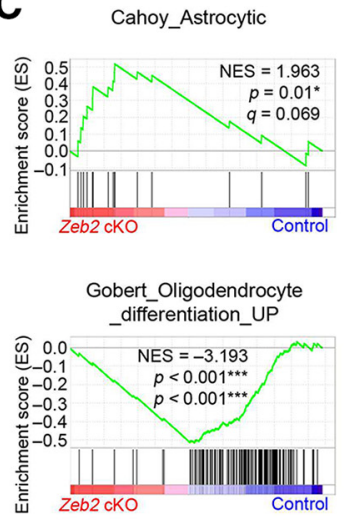

Epithelial_mesenchymal_transition

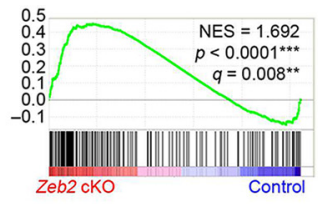

Cell_cycle_mitotic

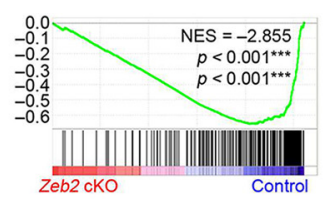

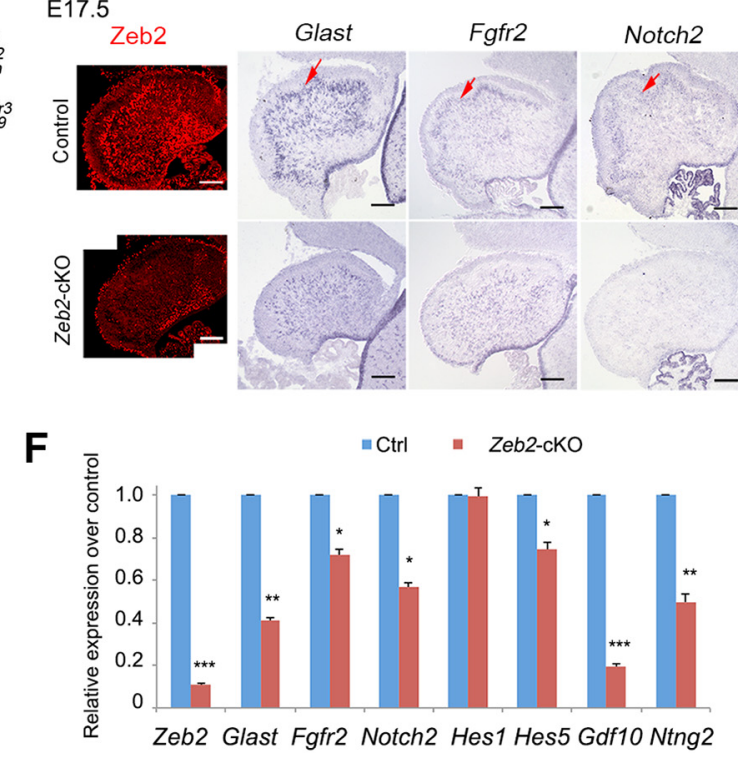

Gdf10

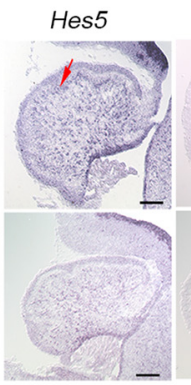

G

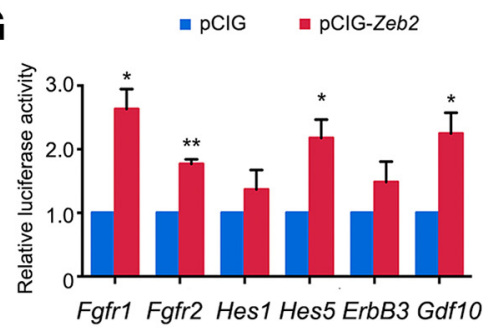

Figure 7. ZEB2 regulates multiple signaling pathways necessary for Bergmann glial development. $A$, Principal component analysis showing separation of gene expression patterns in the cerebellum of heterozygous control (Zeb2 ${ }^{f /+}$ : hGFAP-Cre) and Zeb2-cK0 groups. B, GSEA enrichment analysis of RNA-seq comparing Zeb2-cKO $(n=3)$ and control cerebella ( $\left.n=3\right)$ for gene signatures from the MSigDB database. Top five gene sets upregulated in Zeb2-CKO with FDR $q$ values $<0.03$ are plotted as a function of NESs. Circle size is proportional to the number of significant genes defined here as the number of genes represented in the leading-edge subset, i.e., the subset of members within a gene set that shows statistically significant, concordant differences between two biological states and contribute most to the NES. Circle colors represent FDR q values. C, GSEA enrichment plots show normalized gene expression of representative upregulated gene signatures (astrocytic and EMT signatures) and downregulated gene signatures (oligodendrocyte differentiation and cell cycle signatures) in Zeb2-cKO cerebella compared with control. D, Heat map showing representative Zeb2-regulated genes in control and Zeb2-cK0 mutant cerebella at PO. E, Zeb2 immunostaining and in situ hybridization for Glast, Fgfr2, Hes5, Notch2, Gdf10, and Ntng2 in cerebella from control and Zeb2-cKO mice at E17.5. Arrows indicate the presumptive Bergmann glia layer. Scale bars, $50 \mu \mathrm{m}$. $\boldsymbol{F}$, Quantitative real-time PCR analysis of the indicated genes in control and Zeb2-cKO cerebella at PO ( $n=3$ animals/genotype). Data represent means \pm SEM. ${ }^{*} p<0.05,{ }^{* *} p<0.01$, ${ }^{* * *} p<0.001$. Student's $t$ test. G, Luciferase activity of the reporters driven by ZEB2 consensus sites containing promoter regions of Fgfr 1, Fgfr2, Hes1, Hes5, erbB3, and Gdf10 in $293 \mathrm{~T}$ cells cotransfected with control vector pCIG or vectors for Zeb2 expression. Values presented are the average of three independent experiments. ${ }^{*} p<0.05 ;{ }^{* *} p<0.01$; Student's $t$ test.

glia/neural progenitors mediated by hGFAP-Cre, suggests that ZEB2 is required for Bergmann glia specification from radial glia in the developing cerebellum.

It is worth noting that GFP-expressing cells from the hGFAPGFP transgenic line label specifically astrocytes and Bergmann glia in the developing cerebellum as previously reported (Su et al., 2004; Koirala and Corfas, 2010), they are not equivalent to those labeled by hGFAP-Cre transgenic line, where Cre recombination occurs in radial glia or neural progenitors at embryonic stages, which give rise to both glia and neurons (Zhuo et al., 2001). hGFAP-Cre is also shown to mediate floxed allele recombination in astrocytes, Bergmann glia and their progenitors (Komine et al., 2007). In the developing forebrain, ZEB2 is critical for neurogenesis, neuronal migration, and oligodendrogenesis from neural progenitor cells (Seuntjens et al., 2009; McKinsey et al., 2013; van den Berghe et al., 2013; Parthasarathy et al., 2014). Although we cannot completely exclude the possibility that the defects in cortical development lead to impairment of cerebellar development in Zeb2-cKO mice, we show that ZEB2 is expressed in Bergmann glia, and observed a specific loss of Bergman glia, but not granule neurons despite their migration being impaired.

Although suitable specific markers for Bergmann glia precursors are not available at present, the number and the proliferation of glial precursors labeled by GFAP-GFP in the presumptive PCL at embryonic stages is reduced. We do not detect significant alteration of cell death between control and Zeb2 mutant cerebella assayed by activated caspase 3 expression (data not shown). This suggests that ZEB2 may potentially regulate the proliferation of presumptive Bergmann glia precursor. Consistently, transcriptome profiling of Zeb2-cKO cerebellum at $\mathrm{P} 0$ reveals a significant 
downregulation of the genes related to mitotic cell cycle (Fig. 7). The failure in Bergmann glial differentiation likely contributes to severe defects in inward migration of GNPs from EGL to IGL, causing disorganization of the laminar structure of the cerebellar cortex in Zeb2-deficient cerebella.

Myelination defects caused by Zeb2 deletion in oligodendrocyte lineage cells by Olig1-Cre did not cause a defect in Bergmann glia formation, despite the lack of myelinating oligodendrocytes in the white matter of mutant cerebella. Knock-out of Zeb2 in Atoh $1^{+}$GNPs did not affect granule neuron formation and migration during cerebellar development, which is consistent with the lack of ZEB2 expression in GNPs. These observations suggest that defects in Bergmann glia formation in Zeb2-cKO mice was not due to the non-cell autonomous function of ZEB2 in myelination or GNPs. At present, there is no Bergmann glia-specific Cre line; we cannot definitely define the cell-autonomous role of ZEB2 in Bergmann glia formation or maturation. Given that Bergmann glia are derived from the cerebellar radial glia, our data indicate that ZEB2 is expressed in both Bergmann glia and the radial glia/neural progenitors in the cerebellar ventricular zone, and that deletion of Zeb2 in radial glia by hGFAP-Cre leads to defects in Bergmann glia specification.

In Zeb2-cKO animals, cerebellar dysgenesis appears more severe in anterior lobules than posterior lobules. The incomplete deletion of ZEB2 due to potential regional specificity of Cre recombination in the hGFAP-Cre line or distinct requirements of ZEB2 in a specific region of the cerebellum might contribute to the varying severity of the phenotypes across the Zeb2-cKO cerebellum. Alternatively, it is possible that other unidentified factors may compensate for the loss of ZEB2 function in Bergmann glia formation; however, their identity remains to be determined.

In the Zeb2-cKO cerebellum, intriguingly, some migration events occur and the IGL is visible, this suggests that GNPs potentially could migrate to a certain extent without radial processes or use other cellular processes. The mechanisms underlying inward migration of a population of GNPs in the absence of mature Bergmann glia in the Zeb2-mutant cerebellum remain to be determined. Nonetheless, $\mathrm{ZIC1}^{+}$GNPs were dispersed throughout the ML and IGL layers appears to be disorganized in Zeb2-cKO mutant cerebella, suggesting that Bergmann glia are critical for the organized migration of GNPs and proper cerebellar lamination.

Given the defects in Bergmann glia differentiation in Zeb2cKO mutants, downregulated genes may reflect those genes or pathways highly expressed in Bergmann glia. Transcriptome profiling analysis indicated a downregulation of $\mathrm{NOTCH}$ signaling components, including HES5, and FGF signaling such as FGFR2 in the Zeb2-cKO cerebellum. NOTCH signaling components are expressed in Bergmann glia in addition to neural progenitors (Tanaka et al., 1999; Ohtsuka et al., 2001; Koirala and Corfas, 2010; de Oliveira-Carlos et al., 2013). Similarly, FGFR2 is expressed in a spatiotemporal pattern consistent with expression in Bergmann glia (Lin et al., 2009). Given that the mice with deletion of $F g f r 1 / 2$ or $S h p 2$, which is essential for FGF/ERK activation, display a defect in the induction of Bergmann glia formation similar to the Zeb2-cKO mice (Müller Smith et al., 2012; Li et al., 2014), it remains to be determined whether ZEB2 is critical for FGF signaling activation during Bergmann glia development. We find that overexpression of ZEB2 enhanced the promoter activity of FGF receptors Fgfr 1 and Fgfr2, a NOTCH effector Hes5, as well as a TGF $\beta$ receptor ligand $G d f 10$, suggesting that ZEB2 overex- pression may enhance FGF, NOTCH and TGF $\beta$ receptor signaling for Bergmann gliogenesis.

Transcriptome analysis reveals a set of new Bergmann gliaexpressing genes. Consistent with the reduction of Bergmann glia in the Zeb2-cKO cerebellum, we observed a downregulation of axonal guidance factors such as Netrin G2, which regulates attraction and repulsion of neuronal axons (Barallobre et al., 2005; Nishimura-Akiyoshi et al., 2007) and GDF10, which modulates axonal sprouting ( $\mathrm{Li}$ et al., 2015). In contrast to predominant expression of Netrin family members in neurons of other brain regions (Barallobre et al., 2005; Nishimura-Akiyoshi et al., 2007), our data that Ntng2 encoding Netrin G2 and GDF10 are highly enriched in Bergmann glia in the developing cerebellum, it would be interesting to determine whether Bergmann glia-expressing axonal guidance cues are required for GNP migration and Bergmann glial morphogenesis.

Bergmann glia and astrocytes share many common gene expression profiles, intriguingly, we found that Bergmann gliaenriched gene expression was downregulated, whereas a set of astrocytic genes (Cahoy et al., 2008) was upregulated in the Zeb2cKO cerebellum (Fig. 7). GFAP-GFP ${ }^{+} / S 100 \beta^{+}$astrocytes were present in the PCL, where Bergmann glia were lost. This raises a possibility that ZEB2 may regulate the fate switch between Bergmann glia and astrocytes. In the absence of ZEB2, a population of Bergmann glia precursors that fail to differentiate into mature radial process-bearing Bergmann glia might become astrocytes.

Previous efforts to discover the mechanisms that drive cerebellar foliation initiation and gyrification have mostly focused on the role of GNPs and Purkinje cells (Lewis et al., 2004; Swanson et al., 2005; Corrales et al., 2006; Sudarov and Joyner, 2007). Our data show that the Zeb2 loss causes cerebellar foliation defects through regulating Bergmann glia formation, suggesting a critical role of Zeb2-dependent Bergmann glia development for cerebellar foliation and laminar organization. MOWS is an autosomal dominant disorder characterized by a nonrandom association of multiple birth defects impairing normal development and motor deficits. The phenotypic variation between the ZEB2 haploinsufficient human mutants and heterozygous knock-out mice could be due to a sensitivity to different gene dosage between mouse and human or dominant-negative effects of human mutant proteins, which may produce effects similar to homozygous-null Zeb2 in mice. The critical role of ZEB2 in the control of Bergmann glia formation in cerebellar lamination points to a potential mechanism of motor control and coordination in MOWS patients with ZEB2 mutations.

\section{References}

Barallobre MJ, Pascual M, Del Río JA, Soriano E (2005) The netrin family of guidance factors: emphasis on netrin-1 signalling. Brain Res Brain Res Rev 49:22-47. CrossRef Medline

Bellamy TC (2006) Interactions between purkinje neurones and Bergmann glia. Cerebellum 5:116-126. CrossRef Medline

Belvindrah R, Nalbant P, Ding S, Wu C, Bokoch GM, Müller U (2006) Integrinlinked kinase regulates Bergmann glial differentiation during cerebellar development. Mol Cell Neurosci 33:109-125. CrossRef Medline

Boyden ES, Katoh A, Raymond JL (2004) Cerebellum-dependent learning: the role of multiple plasticity mechanisms. Annu Rev Neurosci 27:581609. CrossRef Medline

Buckner RL (2013) The cerebellum and cognitive function: 25 years of insight from anatomy and neuroimaging. Neuron 80:807-815. CrossRef Medline

Cacheux V, Dastot-Le Moal F, Kääriäinen H, Bondurand N, Rintala R, Boissier B, Wilson M, Mowat D, Goossens M (2001) Loss-of-function mu- 
tations in $\operatorname{SIP}_{1}$ Smad interacting protein 1 result in a syndromic Hirschsprung disease. Hum Mol Genet 10:1503-1510. CrossRef Medline

Cahoy JD, Emery B, Kaushal A, Foo LC, Zamanian JL, Christopherson KS, Xing Y, Lubischer JL, Krieg PA, Krupenko SA, Thompson WJ, Barres BA (2008) A transcriptome database for astrocytes, neurons, and oligodendrocytes: a new resource for understanding brain development and function. J Neurosci 28:264-278. CrossRef Medline

Corrales JD, Blaess S, Mahoney EM, Joyner AL (2006) The level of sonic hedgehog signaling regulates the complexity of cerebellar foliation. Development 133:1811-1821. CrossRef Medline

de Oliveira-Carlos V, Ganz J, Hans S, Kaslin J, Brand M (2013) Notch receptor expression in neurogenic regions of the adult zebrafish brain. PLoS One 8:e73384. CrossRef Medline

Eiraku M, Tohgo A, Ono K, Kaneko M, Fujishima K, Hirano T, Kengaku M (2005) DNER acts as a neuron-specific notch ligand during Bergmann glial development. Nat Neurosci 8:873-880. CrossRef Medline

Hatten ME (1999) Expansion of CNS precursor pools: a new role for sonic hedgehog. Neuron 22:2-3. CrossRef Medline

He X, Zhang L, Chen Y, Remke M, Shih D, Lu F, Wang H, Deng Y, Yu Y, Xia Y, Wu X, Ramaswamy V, Hu T, Wang F, Zhou W, Burns DK, Kim SH, Kool M, Pfister SM, Weinstein LS, et al. (2014) The G protein $\alpha$ subunit $\mathrm{G} \alpha_{\mathrm{s}}$ is a tumor suppressor in Sonic hedgehog-driven medulloblastoma. Nat Med 20:1035-1042. CrossRef Medline

Ito M (2006) Cerebellar circuitry as a neuronal machine. Prog Neurobiol 78:272-303. CrossRef Medline

Koirala S, Corfas G (2010) Identification of novel glial genes by single-cell transcriptional profiling of bergmann glial cells from mouse cerebellum. PLoS One 5:e9198. CrossRef Medline

Komine O, Nagaoka M, Watase K, Gutmann DH, Tanigaki K, Honjo T, Radtke F, Saito T, Chiba S, Tanaka K (2007) The monolayer formation of Bergmann glial cells is regulated by Notch/RBP-J signaling. Dev Biol 311:238-250. CrossRef Medline

Lewis PM, Gritli-Linde A, Smeyne R, Kottmann A, McMahon AP (2004) Sonic hedgehog signaling is required for expansion of granule neuron precursors and patterning of the mouse cerebellum. Dev Biol 270:393410. CrossRef Medline

Li K, Leung AW, Guo Q, Yang W, Li JY (2014) Shp2-dependent ERK signaling is essential for induction of Bergmann glia and foliation of the cerebellum. J Neurosci 34:922-931. CrossRef Medline

Li S, Nie EH, Yin Y, Benowitz LI, Tung S, Vinters HV, Bahjat FR, StenzelPoore MP, Kawaguchi R, Coppola G, Carmichael ST (2015) GDF10 is a signal for axonal sprouting and functional recovery after stroke. Nat Neurosci 18:1737-1745. CrossRef Medline

Lin Y, Chen L, Lin C, Luo Y, Tsai RY, Wang F (2009) Neuron-derived FGF9 is essential for scaffold formation of Bergmann radial fibers and migration of granule neurons in the cerebellum. Dev Biol 329:44-54. CrossRef Medline

López-Bayghen E, Rosas S, Castelán F, Ortega A (2007) Cerebellar Bergmann glia: an important model to study neuron-glia interactions. Neuron Glia Biol 3:155-167. CrossRef Medline

Lumpkin EA, Collisson T, Parab P, Omer-Abdalla A, Haeberle H, Chen P, Doetzlhofer A, White P, Groves A, Segil N, Johnson JE (2003) Math1driven GFP expression in the developing nervous system of transgenic mice. Gene Expr Patterns 3:389-395. CrossRef Medline

LütolfS, Radtke F, Aguet M, Suter U, Taylor V (2002) Notchl is required for neuronal and glial differentiation in the cerebellum. Development 129: 373-385. Medline

Malatesta P, Hack MA, Hartfuss E, Kettenmann H, Klinkert W, Kirchhoff F, Götz M (2003) Neuronal or glial progeny: regional differences in radial glia fate. Neuron 37:751-764. CrossRef Medline

McKinsey GL, Lindtner S, Trzcinski B, Visel A, Pennacchio LA, Huylebroeck D, Higashi Y, Rubenstein JL (2013) Dlx1\&2-dependent expression of $Z f h x 1 b$ (Sip1, Zeb2) regulates the fate switch between cortical and striatal interneurons. Neuron 77:83-98. CrossRef Medline

Mecklenburg N, Martinez-Lopez JE, Moreno-Bravo JA, Perez-Balaguer A, Puelles E, Martinez S (2014) Growth and differentiation factor 10 (Gdf10) is involved in Bergmann glial cell development under shh regulation. Glia 62:1713-1723. CrossRef Medline

Millen KJ, Gleeson JG (2008) Cerebellar development and disease. Curr Opin Neurobiol 18:12-19. CrossRef Medline

Mowat DR, Wilson MJ, Goossens M (2003) Mowat-Wilson syndrome. J Med Genet 40:305-310. CrossRef Medline
Müller Smith K, Williamson TL, Schwartz ML, Vaccarino FM (2012) Impaired motor coordination and disrupted cerebellar architecture in Fgfrl and Fgfr2 double knockout mice. Brain Res 1460:12-24. CrossRef Medline

Nishimura-Akiyoshi S, Niimi K, Nakashiba T, Itohara S (2007) Axonal netrin-gs transneuronally determine lamina-specific subdendritic segments. Proc Natl Acad Sci U S A 104:14801-14806. CrossRef Medline

Nishizaki Y, Takagi T, Matsui F, Higashi Y (2014) SIP1 expression patterns in brain investigated by generating a SIP1-EGFP reporter knock-in mouse. Genesis 52:56-67. CrossRef Medline

Ohtsuka T, Sakamoto M, Guillemot F, Kageyama R (2001) Roles of the basic helix-loop-helix genes Hes1 and Hes5 in expansion of neural stem cells of the developing brain. J Biol Chem 276:30467-30474. CrossRef Medline

Parmigiani E, Leto K, Rolando C, Figueres-Oñate M, López-Mascaraque L, Buffo A, Rossi F (2015) Heterogeneity and bipotency of astroglial-like cerebellar progenitors along the interneuron and glial lineages. J Neurosci 35:7388-7402. CrossRef Medline

Parthasarathy S, Srivatsa S, Nityanandam A, Tarabykin V (2014) Ntf3 acts downstream of Sip1 in cortical postmitotic neurons to control progenitor cell fate through feedback signaling. Development 141:3324-3330. CrossRef Medline

Roussel MF, Hatten ME (2011) Cerebellum development and medulloblastoma. Curr Top Dev Biol 94:235-282. CrossRef Medline

Sathyamurthy A, Yin DM, Barik A, Shen C, Bean JC, Figueiredo D, She JX, Xiong WC, Mei L (2015) ERBB3-mediated regulation of Bergmann glia proliferation in cerebellar lamination. Development 142:522-532. CrossRef Medline

Seuntjens E, Nityanandam A, Miquelajauregui A, Debruyn J, Stryjewska A, Goebbels S, Nave KA, Huylebroeck D, Tarabykin V (2009) Sip 1 regulates sequential fate decisions by feedback signaling from postmitotic neurons to progenitors. Nat Neurosci 12:1373-1380. CrossRef Medline

Sillitoe RV, Joyner AL (2007) Morphology, molecular codes, and circuitry produce the three-dimensional complexity of the cerebellum. Annu Rev Cell Dev Biol 23:549-577. CrossRef Medline

Su M, Hu H, Lee Y, d'Azzo A, Messing A, Brenner M (2004) Expression specificity of GFAP transgenes. Neurochem Res 29:2075-2093. CrossRef Medline

Sudarov A, Joyner AL (2007) Cerebellum morphogenesis: the foliation pattern is orchestrated by multi-cellular anchoring centers. Neural Dev 2:26. CrossRef Medline

Swanson DJ, Tong Y, Goldowitz D (2005) Disruption of cerebellar granule cell development in the Pax6 mutant, Sey mouse. Brain Res Dev Brain Res 160:176-193. CrossRef Medline

Tanaka M, Kadokawa Y, Hamada Y, Marunouchi T (1999) Notch2 expression negatively correlates with glial differentiation in the postnatal mouse brain. J Neurobiol 41:524-539. CrossRef Medline

van den Berghe V, Stappers E, Vandesande B, Dimidschstein J, Kroes R, Francis A, Conidi A, Lesage F, Dries R, Cazzola S, Berx G, Kessaris N, Vanderhaeghen P, van Ijcken W, Grosveld FG, Goossens S, Haigh JJ, Fishell G, Goffinet A, Aerts S, et al. (2013) Directed migration of cortical interneurons depends on the cell-autonomous action of Sip1. Neuron 77:70-82. CrossRef Medline

Vandewalle C, Comijn J, De Craene B, Vermassen P, Bruyneel E, Andersen H, Tulchinsky E, Van Roy F, Berx G (2005) SIP1/Zeb2 induces EMT by repressing genes of different epithelial cell-cell junctions. Nucleic Acids Res 33:6566-6578. CrossRef Medline

Verschueren K, Remacle JE, Collart C, Kraft H, Baker BS, Tylzanowski P, Nelles L, Wuytens G, Su MT, Bodmer R, Smith JC, Huylebroeck D (1999) SIP1, a novel zinc finger/homeodomain repressor, interacts with smad proteins and binds to $5^{\prime}$-CACCT sequences in candidate target genes. J Biol Chem 274:20489-20498. CrossRef Medline

Wakamatsu N, Yamada Y, Yamada K, Ono T, Nomura N, Taniguchi H, Kitoh H, Mutoh N, Yamanaka T, Mushiake K, Kato K, Sonta S, Nagaya M (2001) Mutations in SIP1, encoding smad interacting protein-1, cause a form of Hirschsprung disease. Nat Genet 27:369-370. CrossRef Medline Wang VY, Zoghbi HY (2001) Genetic regulation of cerebellar development. Nat Rev Neurosci 2:484-491. CrossRef Medline

Weng Q, Chen Y, Wang H, Xu X, Yang B, He Q, Shou W, Higashi Y, van den Berghe V, Seuntjens E, Kernie SG, Bukshpun P, Sherr EH, Huylebroeck D, Lu QR (2012) Dual-mode modulation of Smad signaling by Smad- 
interacting protein Sip1 is required for myelination in the central nervous system. Neuron 73:713-728. CrossRef Medline

Wu LM, Wang J, Conidi A, Zhao C, Wang H, Ford Z, Zhang L, Zweier C, Ayee BG, Maurel P, Zwijsen A, Chan JR, Jankowski MP, Huylebroeck D, Lu QR (2016) Zeb2 recruits HDAC-NuRD to inhibit notch and controls Schwann cell differentiation and remyelination. Nat Neurosci 19:10601072. CrossRef Medline

Xin M, Yue T, Ma Z, Wu FF, Gow A, Lu QR (2005) Myelinogenesis and axonal recognition by oligodendrocytes in brain are uncoupled in Olig1null mice. J Neurosci 25:1354-1365. CrossRef Medline

Yamada K, Watanabe M (2002) Cytodifferentiation of Bergmann glia and its relationship with purkinje cells. Anat Sci Int 77:94-108. CrossRef Medline
Yuasa S (1996) Bergmann glial development in the mouse cerebellum as revealed by tenascin expression. Anat Embryol (Berl) 194:223-234. Medline

Yue T, Xian K, Hurlock E, Xin M, Kernie SG, Parada LF, Lu QR (2006) A critical role for dorsal progenitors in cortical myelination. J Neurosci 26:1275-1280. CrossRef Medline

Zhuo L, Sun B, Zhang CL, Fine A, Chiu SY, Messing A (1997) Live astrocytes visualized by green fluorescent protein in transgenic mice. Dev Biol 187: 36-42. CrossRef Medline

Zhuo L, Theis M, Alvarez-Maya I, Brenner M, Willecke K, Messing A (2001) hGFAP-Cre transgenic mice for manipulation of glial and neuronal function in vivo. Genesis 31:85-94. CrossRef Medline 